\title{
THE CLASSIFICATION OF HIGHER-ORDER CUSP FORMS
}

\author{
NIKOLAOS DIAMANTIS AND DAVID SIM (UnIVERSITY OF NotTINGHAM) \\ nikolaos.diamantis@nottingham.ac.uk
}

\section{Introduction}

In this paper we establish the complete classification of cusp forms of all orders. Higher-order cusp forms constitute a natural extension of the notion of classical cusp forms and they have attracted the interest of several researchers in the broader area of automorphic forms during the last few years. Reasons for this interest include its relevance for new approaches to problems related to the distribution of modular symbols ([CDO]), to GL(2) $L$-functions ([DKMO], [F]), to percolation theory ([KZ]) and, more recently, to Manin's non-commutative modular symbols ([M]) via the connection of higher-order forms with iterated integrals ([DS]). This approach has already yielded striking successes, e.g. the proof that modular symbols have a normal distribution $([\mathrm{PR}])$, the establishment of higher order Kronecker limit formulas ([JO]) etc.

The first step towards a classification of spaces of higher-order cusp forms was taken in [DO], where the case of order 2 was settled. Unexpectedly, the classification of higher weights proved to be far from routine and it seems that ultimately this is related to difficulties to identify the underlying cohomology in orders higher than 2. Indeed, we have not found yet an Eichler-Shimura-type theorem similar to that proved in [DO]. However, in this work we succeed in computing the dimensions of the spaces of higher-order forms and in constructing explicit bases that are fully computable.

The first two sections deal with technical preliminaries some of which have independent interest (e.g. the growth estimates for antiderivatives of higher-order cusp forms). The basic theorem, establishing the analytic continuation and growth properties of the generalized Poincare series on which the basis elements is built, is proved in section 3.2. It is based on a quite large scale induction which relies crucially on the spectral analysis of a certain Poincaré-type series. The basis elements are constructed in Sections 3.3 and 3.4, where it is also proved that they are actually a basis of the space of weight 2 higher-order cusp forms. In section 4 we contruct bases for higher-order cusp forms of higher weight.

\section{Definitions and basic estimates}

Let $\Gamma \subset P S L_{2}(\mathbb{R})$ be a Fuchsian group of the first kind acting on the upper half plane $\mathfrak{H}$ with non compact quotient $\Gamma \backslash \mathfrak{H}$ of genus $g$. We assume that there are $m \geq 2$ inequivalent cusps. As usual we write $x+i y=z \in \mathfrak{H}$ and $\mathfrak{H}^{*}$ for $\mathfrak{H}$ together with the cusps. Let $d \mu z$ be the hyperbolic volume form $d x d y / y^{2}$ and

$$
V=\int_{\Gamma \backslash \mathfrak{H}} \frac{d x d y}{y^{2}}
$$

the volume of $\Gamma \backslash \mathfrak{H}$. For a fundamental domain $\mathfrak{F}$ fix representatives of the inequivalent cusps in $\overline{\mathfrak{F}}$ and give them labels such as $\mathfrak{a}, \mathfrak{b}$. Use the corresponding scaling matrices $\sigma_{\mathfrak{a}}, \sigma_{\mathfrak{b}}$ to give convenient local coordinates near these cusps as in [I1], Ch 2. The subgroup $\Gamma_{\mathfrak{a}}$ is the set of elements of $\Gamma$ fixing $\mathfrak{a}$ and

$$
\sigma_{\mathfrak{a}}{ }^{-1} \Gamma_{\mathfrak{a}} \sigma_{\mathfrak{a}}=\Gamma_{\infty}=\left\{ \pm\left(\begin{array}{cc}
1 & m \\
0 & 1
\end{array}\right) \mid m \in \mathbb{Z}\right\} .
$$


The slash operator $\left.\right|_{k}$ defines an action of $P S L_{2}(\mathbb{R})$ on functions $f: \mathfrak{H} \mapsto \mathbb{C}$ by

$$
\left(\left.f\right|_{k} \gamma\right)(z)=f(\gamma z)(c z+d)^{-k}
$$

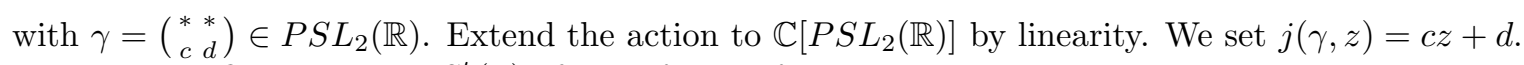

We now define the space $S_{k}^{t}(\Gamma)$ of cusp forms of order $t$ and weight $k \geq 0$ recursively by setting:

(i) $S_{k}^{0}(\Gamma)=\{0\}$ and

(ii) for $t \geq 1$, by letting $S_{k}^{t}(\Gamma)$ be the space of holomorphic functions $f: \mathfrak{H} \rightarrow \mathbb{C}$ such that:

1. $\left.f\right|_{k}(\gamma-1) \in S_{k}^{t-1}(\Gamma)$, for all $\gamma \in \Gamma$,

2. $\left.f\right|_{k} \pi=f$, for all parabolic $\pi \in \Gamma$ and

3. for each cusp $\mathfrak{a},\left(\left.f\right|_{k} \sigma_{\mathfrak{a}}\right)(z) \ll e^{-c y}$ as $y \rightarrow \infty$ uniformly in $x$ for some constant $c>0$ ("vanishing at the cusps").

When the group is clear, we will be using $S_{k}^{t}$ instead of $S_{k}^{t}(\Gamma)$.

A useful reformulation of this definition, essentially proved in [DKMO] is that a holomorphic $f: \mathfrak{H} \rightarrow \mathbb{C}$ is a $t$-th order cusp form if and only if it is invariant under the parabolic elements, it satisfies

$$
\left.f\right|_{k}\left(\gamma_{1}-1\right) \ldots\left(\gamma_{t}-1\right)=0 \text { for all } \gamma_{i} \in \Gamma
$$

and, for each $\gamma \in \Gamma$ and each cusp $\mathfrak{a},\left(\left.f\right|_{k} \gamma \sigma_{\mathfrak{a}}\right)(z) \ll e^{-c y}$ as $y \rightarrow \infty$ uniformly in $x$ with $c>0$ and the implied constant depending on $\gamma$.

Further, by relaxing the third condition to include functions such that, for each cusp $\mathfrak{a},\left(\left.f\right|_{k} \sigma_{\mathfrak{a}}\right)(z) \ll$ $y^{c}$ as $y \rightarrow \infty$ uniformly in $x$ for some constant $c$, we obtain the space of $t$-th order modular forms. We denote it by $M_{k}^{t}$.

The next lemma is stated in greater generality than what we need it for in the sequel because we want it to cover other situations that have arisen in our work.

We define recursively certain sets of maps denoted by $\mathcal{H}_{t}$. First, for convenience we use the superscripts ${ }^{+}$and ${ }^{-}$to indicate absence and presence of complex conjugation respectively. We set

(i) $\mathcal{H}_{0}=\mathcal{H}_{1}=\{0\}$ and

(ii) for $t>1$, we set

$$
\mathcal{H}_{t}:=\bigoplus_{\substack{r+s \leq t \\ 1 \leq r, s \leq t-1}} H_{r} \otimes S_{2}^{s}
$$

where $H_{r} \subset \operatorname{Maps}(\Gamma, \mathbb{C})$ is generated by maps of the form

$$
\gamma \rightarrow \prod_{i=1}^{m}\left(\int_{z_{i}}^{\gamma z_{i}} f_{i}(w) d w\right)^{ \pm}
$$

for some $z_{i} \in \mathfrak{H}^{*}$ with $f_{i} \in S_{2}^{r_{i}}$ such that $\left.f_{i}\right|_{2}(\cdot-1) \in \mathcal{H}_{r_{i}}$ and $\sum_{i=1}^{m} r_{i}=r$. (Here and in the sequel we will take sums whose upper limit is smaller than the lower to be equal to 0 .) We identify each of these spaces with their images in $\operatorname{Maps}\left(\Gamma, S_{2}^{t-1}\right)$ under the natural projection.

With this notation we can now state a proposition that gives important estimates for derivatives and anti-derivatives of weight 2 cusp forms of all orders.

Lemma 2.1. For all $t \geq 0$, if $f \in S_{2}^{t}$ satisfies $\left.f\right|_{2}(\cdot-1) \in \mathcal{H}_{t}$ then, for any cusp $\mathfrak{a}$,

$$
\begin{aligned}
& \text { (i) } \operatorname{Im}(z)|f(z)| \ll \sum_{i=0}^{t-1}|\log (\operatorname{Im}(z))|^{i} \\
& \text { (ii) } \int_{z_{0}}^{z} f(w) d w \ll \sum_{j=0}^{t}\left|\log \left(\operatorname{Im}\left({\sigma_{\mathfrak{a}}}^{-1} z\right)\right)\right|^{j} \quad \text { and } \\
& \text { (iii) } \int_{z_{0}}^{\gamma z_{0}} f(w) d w \ll \sum_{\substack{i+j \leq t \\
0 \leq i, j}}\left|\log \left(\operatorname{Im}\left(\sigma_{\mathfrak{a}}{ }^{-1} z\right)\right)\right|^{i}\left|\log \left(\operatorname{Im}\left(\sigma_{\mathfrak{a}}{ }^{-1} \gamma z\right)\right)\right|^{j} \\
& 2
\end{aligned}
$$


for all $z \in \mathfrak{H}$. The implied constants are independent of $z$ and $\gamma$.

Proof: We will use induction on $t$. For $t=0$, it is trivial. Let now $t>0$. Assume the result holds for orders $<t$ and let $f \in S_{2}^{t}$ satisfy $\left.f\right|_{2}(\cdot-1) \in \mathcal{H}_{t}$. To prove (i), let $F_{\infty}$ be the strip of $(x, y)$ with $y>0$ and $|x| \leq 1 / 2$ and $F$ the fundamental domain consisting of $z \in F_{\infty}$ such that $|j(\gamma, z)|>1$ for all $\gamma \in \Gamma-\Gamma_{\infty}$. Then $\operatorname{Im}(z)|f(z)| \ll 1$ in $F$ because $f$ has exponential decay at each cusp. If, on the other hand, $z \in F_{\infty}-F$, then there is $\gamma \in \Gamma-\Gamma_{\infty}$ and $w \in F$ such that $z=\gamma w$. According to Lemma 1.25 of [Sh], there is a $r>0$ depending only on $\Gamma$ such that $\operatorname{Im}(w) \leq 1 /\left(r^{2} \operatorname{Im}(z)\right)$. On the other hand, since $w \in F, \operatorname{Im}(z)<\operatorname{Im}(w)$. Therefore, $\log (\operatorname{Im}(z))<\log (\operatorname{Im}(w)) \leq-2 \log (r)-\log (\operatorname{Im}(z))$ and hence

$$
|\log (\operatorname{Im}(w))| \leq 2|\log (r)|+|\log (\operatorname{Im}(z))| .
$$

Now, if

$$
\left(\left.f\right|_{2}(\gamma-1)\right)(z)=\sum \prod_{i=1}^{m}\left(\int_{z_{i}}^{\gamma z_{i}} h_{i}(w) d w\right)^{ \pm} h_{0}(z)
$$

where the prime indicates that the summation is over $\left(h_{1}, \ldots, h_{m}, h_{0}\right) \in S_{2}^{l_{1}} \times \cdots \times S_{2}^{l_{m}} \times S_{2}^{l_{0}}\left(l_{1}+\right.$ $\left.\cdots+l_{m}+l_{0} \leq t\right)$ with $\left.h_{i}\right|_{2}(\cdot-1) \in \mathcal{H}_{l_{i}}(i>0)$, then

$$
|\operatorname{Im}(z) f(z)|=\left|\operatorname{Im}(w)\left(\left.f\right|_{2} \gamma\right)(w)\right| \leq|\operatorname{Im}(w) f(w)|+\sum^{\prime}\left|\prod_{i=1}^{m} \int_{z_{i}}^{\gamma z_{i}} h_{i}(w) d w\right|\left|\operatorname{Im}(w) h_{0}(w)\right| .
$$

With the boundedness of $\operatorname{Im}(z)|f(z)|$ in $F$ and the inductive hypothesis, we deduce that

$$
|\operatorname{Im}(z) f(z)| \ll 1+\sum^{\prime} \prod_{n=1}^{m}\left(\sum_{\substack{i+j \leq l_{n} \\ 0 \leq i, j}}|\log (\operatorname{Im}(w))|^{i}|\log (\operatorname{Im}(\gamma w))|^{j}\right)\left(\sum_{i=0}^{l_{0}-1}|\log (\operatorname{Im}(w))|^{i}\right)
$$

with the implied constant independent of $w$ and $\gamma$. With (2.1) this implies

$$
|\operatorname{Im}(z) f(z)| \ll 1+\sum^{\prime} \prod_{n=1}^{m}\left(\sum_{\substack{i+j \leq l_{n} \\ 0 \leq i, j}}|\log (\operatorname{Im}(z))|^{i+j}\right)\left(\sum_{i=0}^{l_{0}-1}|\log (\operatorname{Im}(z))|^{i}\right) \ll \sum_{i=0}^{t-1}|\log (\operatorname{Im}(z))|^{i}
$$

for $z \in F_{\infty}-F$ and thus for all $z \in \mathfrak{H}$ because both sides of (i) are translation invariant.

To prove (ii), we first note that

$$
\int_{z_{0}}^{\sigma_{\mathfrak{a}} z} f(w) d w=\int_{\sigma_{\mathfrak{a}}-1}^{z}\left(\left.f\right|_{2} \sigma_{\mathfrak{a}}\right)(w) d w .
$$

Since $\left.f\right|_{2} \sigma_{\mathfrak{a}}\left(\sigma_{\mathfrak{a}}{ }^{-1} \gamma \sigma_{\mathfrak{a}}-1\right)=\left.f\right|_{2}(\gamma-1) \sigma_{\mathfrak{a}}$, an inductive argument implies that $\left.f\right|_{2} \sigma_{\mathfrak{a}} \in S_{2}^{t}\left(\Gamma^{\prime}\right)$ with $\Gamma^{\prime}:=\sigma_{\mathfrak{a}}^{-1} \Gamma \sigma_{\mathfrak{a}}$. In fact, a similar argument implies that $\left.f\right|_{2} \sigma_{\mathfrak{a}}(\cdot-1) \in \mathcal{H}_{t}\left(\Gamma^{\prime}\right)$. Therefore, we can use (i) to deduce

$$
\operatorname{Im}(z)\left|\left(\left.f\right|_{2} \sigma_{\mathfrak{a}}\right)(z)\right| \ll \sum_{i=0}^{t-1}|\log (\operatorname{Im}(z))|^{i}
$$

Further the invariance under the parabolic elements gives

$$
\int_{z}^{z+1}\left(\left.f\right|_{2} \sigma_{\mathfrak{a}}\right)(w) d w=0 .
$$

Therefore,

$$
\int_{z_{0}}^{\sigma_{\mathfrak{a}} z} f(w) d w \ll \int_{\sigma_{\mathfrak{a}}^{-1} z_{0}}^{x+i y} \frac{\log (\operatorname{Im} w)^{t-1}+\cdots+1}{\operatorname{Im}(w)} d w
$$


with $y=\operatorname{Im} z$ and $x \equiv \operatorname{Re} z \bmod 1$ and $0 \leq x<1$. The last integral equals

$$
\int_{\sigma_{\mathfrak{a}}^{-1} z_{0}}^{x+i \operatorname{Im}\left({\sigma_{\mathfrak{a}}}^{-1} z_{0}\right)}+\int_{x+i \operatorname{Im}\left(\sigma_{\mathfrak{a}}{ }^{-1} z_{0}\right)}^{x+i y} \frac{\log (\operatorname{Im} w)^{t-1}+\cdots+1}{\operatorname{Im}(w)} d w
$$

where the integration path is, in both cases, a straight segment. This sum in turn equals:

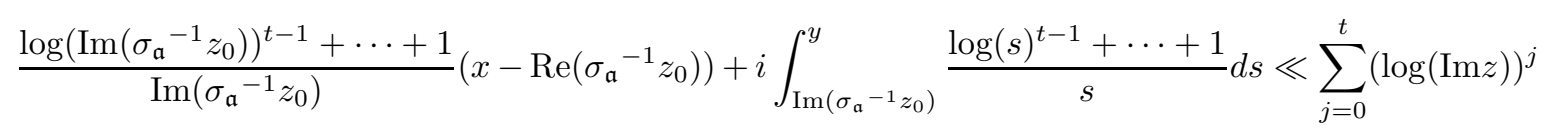

Replacing $z$ by $\sigma_{\mathfrak{a}}^{-1} z$ completes the proof of (ii).

Finally, by employing (2.2) it is easy to see that

$$
\int_{z_{0}}^{\gamma z_{0}} f(w) d w=\int_{z_{0}}^{\gamma z} f(w) d w-\int_{z_{0}}^{z} f(w) d w+\sum \prod_{i=1}^{m}\left(\int_{z_{i}}^{\gamma z_{i}} h_{i}(w) d w\right)^{ \pm} \int_{z}^{z_{0}} h_{0}(w) d w
$$

where the summation is over $\left(h_{1}, \ldots, h_{m}, h_{0}\right) \in S_{2}^{l_{1}} \times \cdots \times S_{2}^{l_{m}} \times S_{2}^{l_{0}},\left(l_{1}+\cdots+l_{m}+l_{0} \leq t\right)$ with $\left.h_{i}\right|_{2}(\cdot-1) \in \mathcal{H}_{l_{i}},(i>0)$ and the inequality follows from (ii) and the inductive hypothesis.

\section{Bases for $S_{2}^{t}(\Gamma)$}

\subsection{Preliminaries}

We collect here some notation and results we will be using frequently in the sequel.

Let $C^{\infty}(\Gamma \backslash \mathfrak{H}, k)$ denote the space of smooth functions $\psi$ on $\mathfrak{H}$ that transform as

$$
\psi(\gamma z)=\varepsilon(\gamma, z)^{k} \psi(z)
$$

for $\gamma$ in $\Gamma$ and $\varepsilon(\gamma, z)=j(\gamma, z) /|j(\gamma, z)|$. Note that this notion of weight in general differs from the previous definition of weight.

We define the Maass raising and lowering operators by

$$
R_{k}=2 i y \frac{d}{d z}+\frac{k}{2}, L_{k}=-2 i y \frac{d}{d \bar{z}}-\frac{k}{2} .
$$

It is an elementary exercise to show that

$$
R_{k}: C^{\infty}(\Gamma \backslash \mathfrak{H}, k) \rightarrow C^{\infty}(\Gamma \backslash \mathfrak{H}, k+2), L_{k}: C^{\infty}(\Gamma \backslash \mathfrak{H}, k) \rightarrow C^{\infty}(\Gamma \backslash \mathfrak{H}, k-2)
$$

For $n>0$, we write $R^{n}$ for $R_{k+2 n-2} \cdots R_{k+2} R_{k}$ and $L^{n}$ for $L_{k-2 n+2} \cdots L_{k-2} L_{k}$. We also let $L^{0}$ and $R^{0}$ be the identity operator.

A very useful fact proved in [JO] (Lemma 9.2) is that if $\gamma \in \mathrm{PSL}_{2}(\mathbb{R})$ and

$$
\mu(s, k, F):=F(\gamma) \operatorname{Im}(\gamma z)^{s} e(m \gamma z) \varepsilon(\gamma, z)^{-k}
$$

then

$$
\begin{aligned}
& R_{k} \mu(s, k, F)=2 i \mu\left(s+1, k+2, \frac{d}{d z} F\right)+\left(s+\frac{k}{2}\right) \mu(s, k+2, F)-4 \pi m \mu(s+1, k+2, F) \\
& L_{k} \mu(s, k, F)=-2 i \mu\left(s+1, k-2, \frac{d}{d \bar{z}} F\right)+\left(s-\frac{k}{2}\right) \mu(s, k-2, F)
\end{aligned}
$$


The hyperbolic Laplacian $\Delta=-4 y^{2} d / d z d / d \bar{z}$ can be realized as $\Delta=-L_{2} R_{0}=-R_{-2} L_{0}$. Further, for $\tau \in P S L_{2}(\mathbb{R})$, we let the operator $\theta_{\tau, k}: C^{\infty}(\Gamma \backslash \mathfrak{H}, k) \rightarrow C^{\infty}\left(\tau^{-1} \Gamma \tau \backslash \mathfrak{H}, k\right)$ be defined by

$$
\theta_{\tau, k} \psi(z)=\frac{\psi(\tau z)}{\varepsilon(\tau, z)^{k}}
$$

It is easy to verify that this action commutes with the raising and lowering operators:

$$
\begin{aligned}
& \theta_{\tau, k-2} L_{k}=L_{k} \theta_{\tau, k}, \\
& \theta_{\tau, k+2} R_{k}=R_{k} \theta_{\tau, k} .
\end{aligned}
$$

In stating our bounds, the notation $y_{\Gamma}(z)=\max _{\mathfrak{a}}\left(\max _{\gamma \in \Gamma}\left(\operatorname{Im}\left(\sigma_{\mathfrak{a}}{ }^{-1} \gamma z\right)\right)\right)$ will often be useful. For example, if $|\psi|$ is smooth with weight 0 then we write $\psi(z) \ll y_{\Gamma}(z)^{A}$, instead of $\psi\left(\sigma_{\mathfrak{a}} z\right) \ll y^{A}$ for each cusp $\mathfrak{a}$ as $y \rightarrow \infty$. We also use the notation $y_{\mathfrak{F}}(z)=\max _{\mathfrak{a}}\left(\operatorname{Im}\left(\sigma_{\mathfrak{a}}{ }^{-1} z\right)\right)$ for $z$ in a fundamental domain $\mathfrak{F}$.

We next recall that the usual non-holomorphic Eisenstein series

$$
E_{\mathfrak{a}}(z, s)=\sum_{\gamma \in \Gamma_{\mathfrak{a}} \backslash \Gamma} \operatorname{Im}\left({\sigma_{\mathfrak{a}}}^{-1} \gamma z\right)^{s}
$$

is absolutely convergent for $s$ with $\operatorname{Re}(s)>1$ (and uniformly convergent for $s$ in compact sets there) and that it has the Fourier expansion at the cusp $\mathfrak{b}$

$$
\begin{aligned}
E_{\mathfrak{a}}\left(\sigma_{\mathfrak{b}} z, s\right) & =\delta_{\mathfrak{a} \mathfrak{b}} y^{s}+\phi_{\mathfrak{a} \mathfrak{b}}(s) y^{1-s}+\sum_{m \neq 0} \phi_{\mathfrak{a} \mathfrak{b}}(m, s) W_{s}(m z) \\
& =\delta_{\mathfrak{a} \mathfrak{b}} y^{s}+\phi_{\mathfrak{a} \mathfrak{b}}(s) y^{1-s}+O\left(e^{-2 \pi y}\right)
\end{aligned}
$$

as $y \rightarrow \infty$ with an implied constant depending only on $s$ and $\Gamma$.

The hyperbolic Laplacian operates on $L^{2}(\Gamma \backslash \mathfrak{H})$ the space of smooth, automorphic, square integrable functions. Any element $\xi$ of $L^{2}(\Gamma \backslash \mathfrak{H})$ may be expanded according to the discrete and continuous spectrum of $\Delta$ (Roelcke-Selberg decomposition):

$$
\xi(z)=\sum_{j=0}^{\infty}\left\langle\xi, \eta_{j}\right\rangle \eta_{j}(z)+\frac{1}{4 \pi} \sum_{\mathfrak{b}} \int_{-\infty}^{\infty}\left\langle\xi, E_{\mathfrak{b}}(\cdot, 1 / 2+i r)\right\rangle E_{\mathfrak{b}}(z, 1 / 2+i r) d r
$$

where $\left\{\eta_{j}\right\}$ denotes a complete orthonormal basis of Maass forms, with corresponding eigenvalues $\lambda_{j}=s_{j}\left(1-s_{j}\right)$, which forms the discrete spectrum. As always, we will write $s_{j}=\sigma_{j}+i t_{j}$, chosen so that $\sigma_{j} \geqslant 1 / 2$ and $t_{j} \geqslant 0$, and we enumerate the eigenvalues, counted with multiplicity, by $0=\lambda_{0}<\lambda_{1} \leqslant \lambda_{2} \leqslant \cdots$. Weyl's law ((11.3) of [I1]) implies

$$
\#\left\{j|| \lambda_{j} \mid \leqslant T\right\} \ll T .
$$

The decomposition (3.3) is absolutely convergent for each fixed $z$ and uniform on compact subsets of $\mathfrak{H}$, provided $\xi$ and $\Delta \xi$ are smooth and bounded (see, for example, Th. 4.7 and Th. 7.3 of [I1]).

For each $j$, the Fourier expansion of $\eta_{j}$ is

$$
\eta_{j}\left(\sigma_{\mathfrak{a}} z\right)=\rho_{\mathfrak{a} j}(0) y^{1-s_{j}}+\sum_{m \neq 0} \rho_{\mathfrak{a} j}(m) W_{s_{j}}(m z)
$$

For all but finitely many of the $j$ (corresponding to $\lambda_{j}<1 / 4$ ) we have $\sigma_{j}=1 / 2$ and $\rho_{\mathfrak{a} j}(0)=0$. The constant $\delta_{\Gamma}$ used throughout this paper is chosen so that $1-\delta_{\Gamma}>\sigma_{1} \geqslant 1 / 2$.

With this notation we now state 
Lemma 3.1. For all $z \in \mathfrak{H}, T \in \mathbb{R}$ and $n \in \mathbb{Z}_{+}$we have

$$
\begin{aligned}
& \text { (i) } R^{n}\left(\eta_{j}(z)\right), L^{n}\left(\eta_{j}(z)\right) \ll_{\Gamma, n}\left(\left|t_{j}\right|^{n}+1\right) y_{\Gamma}(z)^{1 / 2}+\left(\left|t_{j}\right|^{2 n+5}+1\right) y_{\Gamma}(z)^{-3 / 2} \\
& \text { (ii) } E_{\mathfrak{a}}(z, 1 / 2+i r) \underset{\Gamma, T}{\ll} y_{\Gamma}(z)^{1 / 2} \text { for all } r \in[T, T+1] \\
& \text { (iii) } \int_{T}^{T+1}\left|R^{n} E_{\mathfrak{a}}(z, 1 / 2+i r)\right|^{2} d r, \quad \int_{T}^{T+1}\left|L^{n} E_{\mathfrak{a}}(z, 1 / 2+i r)\right|^{2} d r \ll T^{4 n+12} y_{\Gamma}(z)
\end{aligned}
$$

Proof: For a proof of see Lemma 8.2, (11.12) and Lemma 8.3 of [DO] respectively.

We now define the basic auxiliary functions we will be using in the sequel and prove their basic properties. For $k \in 2 \mathbb{Z}$, we consider

$$
U_{\mathfrak{a} m}(z, s, k)=\sum_{\gamma \in \Gamma_{\mathfrak{a}} \backslash \Gamma} \operatorname{Im}\left(\sigma_{\mathfrak{a}}{ }^{-1} \gamma z\right)^{s} e\left(m \sigma_{\mathfrak{a}}{ }^{-1} \gamma z\right) \varepsilon\left(\sigma_{\mathfrak{a}}{ }^{-1} \gamma, z\right)^{-k}
$$

and for simplicity we set $U_{\mathfrak{a} m}(z, s):=U_{\mathfrak{a} m}(z, s, 0)$. By a direct computation based on (3.1)

$$
\begin{aligned}
& R_{k} U_{\mathfrak{a} m}(z, s, k)=(s+k / 2) U_{\mathfrak{a} m}(z, s, k+2)-4 \pi m U_{\mathfrak{a} m}(z, s+1, k+2) \\
& L_{k} U_{\mathfrak{a} m}(z, s, k)=(s-k / 2) U_{\mathfrak{a} m}(z, s, k-2) .
\end{aligned}
$$

Proposition 3.2. For $k \in 2 \mathbb{Z}, U_{\mathfrak{a} m}(z, s, k)$ has a meromorphic continuation to all $s$ with $R e(s)>$ $1-\delta_{\Gamma}$ its only pole appearing at $s=1$ when $m=k=0$. It is simple with residue $1 / V$. Furthermore,

$$
U_{\mathfrak{a} 0}(z, s, k) \ll y_{\Gamma}(z)^{\sigma} \quad \text { and } U_{\mathfrak{a} m}(z, s, k) \ll y_{\Gamma}(z)^{1 / 2} \quad(m>0)
$$

for these $s$ with the implied constant depending on $s, m, k, \Gamma$.

Proof: This is the content of Propositions B and C of [DO].

Given this analytic continuation we set

$$
P_{\mathfrak{a} m}(z)_{2}:=y^{-1} U_{\mathfrak{a} m}(z, 1,2),
$$

These series are holomorphic for $m>0$ and span $S_{2}(\Gamma)$ (cf. [JO] Th. 3.2). When $m=0$ they satisfy

$$
j\left(\sigma_{\mathfrak{b}}, z\right)^{-2} P_{\mathfrak{a} 0}\left(\sigma_{\mathfrak{b}} z\right)_{2}=\delta_{\mathfrak{a} \mathfrak{b}}-\frac{1}{y V}+O\left(e^{-2 \pi y}\right) \quad \text { as } y \rightarrow \infty
$$

and

$$
y^{2} \frac{d}{d \bar{z}} P_{\mathfrak{a} 0}(z)_{2}=\frac{i}{2 V}
$$

For $f \in S_{2}^{t}$ and $n \in \mathbb{Z}_{\geq 1}$ we set:

$$
I_{\mathfrak{a} n}\left(z_{n}\right)=\int_{i \infty}^{z_{n}} \cdots \int_{i \infty}^{z_{2}} \int_{i \infty}^{z_{1}} f_{\mathfrak{a}}\left(z_{0}\right) d z_{0} d z_{1} \cdots d z_{n-1}
$$

where $f_{\mathfrak{a}}(z)=f\left(\sigma_{\mathfrak{a}} z\right) / j\left(\sigma_{\mathfrak{a}}, z\right)^{2}$. For $n \leq 0$, we set $I_{\mathfrak{a} n}(z)=f_{\mathfrak{a}}^{(-n)}(z)$. We observe that $I_{\mathfrak{a} 1}\left(\sigma_{\mathfrak{a}}{ }^{-1} z\right)=$ $\int_{\mathfrak{a}}^{z} f(w) d w$. With this notation, we set, for each $r \in \mathbb{Z}_{\geq 0}$

$$
Q_{\mathfrak{a} m}(z, s, n, r ; \bar{f})=\sum_{\gamma \in \Gamma_{\mathfrak{a}} \backslash \Gamma} \overline{I_{\mathfrak{a} n}\left(\sigma_{\mathfrak{a}}{ }^{-1} \gamma z\right)} \operatorname{Im}\left({\sigma_{\mathfrak{a}}}^{-1} \gamma z\right)^{s} e\left(m \sigma_{\mathfrak{a}}{ }^{-1} \gamma z\right) \varepsilon\left(\sigma_{\mathfrak{a}}{ }^{-1} \gamma, z\right)^{-r} .
$$

In this section we will give the domains of initial convergence and bounds of this series and its derivative. Their analytic continuation will be discussed in the next section. 
Proposition 3.3. Let $f \in S_{2}^{t}$ be such that $\left.f\right|_{2}(\cdot-1) \in \mathcal{H}_{t}$. For $k \in 2 \mathbb{Z}$ and $\sigma=\operatorname{Re}(s)>1$ the series $Q_{\mathfrak{a} m}(z, s, 1, k, \bar{f})$ and $Q_{\mathfrak{a} m}^{\prime}(z, s, 1, k, \bar{f})$ converge absolutely and uniformly on compacta to analytic functions of $s$. For these $s$ and for all $m \geq 0, Q_{\mathfrak{a} m}(z, s, 1, k ; \bar{f})$ and $(|m|+1)^{-1} y Q_{\mathfrak{a} m}^{\prime}(z, s, 1, k ; \bar{f})$ are bounded by a constant times $y_{\Gamma}(z)^{1 / 2-\sigma / 2}$. The implied constants are independent of $z$ and $m$.

Proof: Let $f \in S_{2}^{t}$ be such that $\left.f\right|_{2}(\cdot-1) \in \mathcal{H}_{t}$. By Lemma 2.1 and the elementary inequality $|\log y|<\epsilon\left(y^{\epsilon}+y^{-\epsilon}\right)$ we have

$$
\int_{\mathfrak{a}}^{\sigma_{\mathfrak{b}} z} f(w) d w \ll \sum_{j=-t}^{t} y^{j \epsilon}
$$

for all $z$ in $\mathfrak{H}$ and hence

$$
\int_{\mathfrak{a}}^{\gamma \sigma_{\mathfrak{b}} z} f(w) d w=\int_{\mathfrak{a}}^{\sigma_{\mathfrak{a}}{\sigma_{\mathfrak{a}}}^{-1} \gamma \sigma_{\mathfrak{b}} z} f(w) d w \ll \sum_{j=-t}^{t} \operatorname{Im}\left({\sigma_{\mathfrak{a}}}^{-1} \gamma \sigma_{\mathfrak{b}} z\right)^{j \epsilon}
$$

for any cusp $\mathfrak{b}$ and any $z$ in $\mathfrak{H}$. The implied constant depends solely on $\mathfrak{a}, \epsilon, f$ and $\Gamma$. Further, the Fourier expansion of $\int_{\mathfrak{b}}^{\sigma \mathfrak{b} z} f(w) d w$ yields

$$
\int_{\mathfrak{a}}^{\sigma_{\mathfrak{b}} z} f(w) d w=\int_{\mathfrak{a}}^{\mathfrak{b}} f(w) d w+\frac{1}{2 \pi i} \sum_{n=1}^{\infty} \frac{a_{\mathfrak{b}}(n)}{n} e(n z)
$$

with $a_{\mathfrak{b}}(n)$ the $n$-th Fourier coefficient of $f$ at the cusp $\mathfrak{b}$. Thus,

$$
\int_{\mathfrak{a}}^{\sigma_{\mathfrak{a}} z} f(w) d w \ll e^{-2 \pi y} \text { as } y \rightarrow \infty .
$$

Consequently, since $\left|e\left(m \sigma_{\mathfrak{a}}{ }^{-1} \gamma z\right) \varepsilon\left(\sigma_{\mathfrak{a}}{ }^{-1} \gamma, z\right)^{-k}\right| \leq 1$,

$$
\begin{gathered}
Q_{\mathfrak{a} m}\left(\sigma_{\mathfrak{a}} z, s, 1, k ; \bar{f}\right) \ll \sum_{\gamma \in \Gamma_{\mathfrak{a}} \backslash \Gamma}\left|\int_{\mathfrak{a}}^{\gamma \sigma_{\mathfrak{a}} z} f(w) d w\right| \operatorname{Im}\left(\sigma_{\mathfrak{a}}{ }^{-1} \gamma \sigma_{\mathfrak{a}} z\right)^{\sigma} \\
\ll e^{-2 \pi y} y^{\sigma}+\sum_{\substack{\gamma \in \Gamma_{\mathfrak{a}} \backslash \Gamma \\
\gamma \neq I}}\left(\sum_{j=-t}^{t} \operatorname{Im}\left(\sigma_{\mathfrak{a}}{ }^{-1} \gamma \sigma_{\mathfrak{a}} z\right)^{\sigma+j \epsilon}\right) \ll y^{1-\sigma+t \epsilon}
\end{gathered}
$$

for $\sigma>1+t \epsilon$ as $y \rightarrow \infty$ by (3.2). When $\mathfrak{a} \neq \mathfrak{b}$ we have

$$
Q_{\mathfrak{a} m}\left(\sigma_{\mathfrak{b}} z, s, 1, k ; \bar{f}\right) \ll \sum_{j=-t}^{t} E_{\mathfrak{a}}\left(\sigma_{\mathfrak{b}} z, \sigma+j \epsilon\right) \ll y^{1-\sigma+t \epsilon}
$$

for $\sigma>1+t \epsilon$ as $y \rightarrow \infty$. Choose $\epsilon=(\sigma-1) / 2 t$ for simplicity and we have demonstrated that

$$
Q_{\mathfrak{a} m}(z, s, 1, k ; \bar{f}) \ll y_{\Gamma}(z)^{1 / 2-\sigma / 2}
$$

for $\sigma>1$ and an implied constant depending on $\sigma, \mathfrak{a}, f$ and $\Gamma$ alone. This proves the statement for $Q_{\mathfrak{a} m}(z, s, 1, k ; \bar{f})$.

With (3.1) we deduce that

$$
\begin{aligned}
& 2 i y Q_{\mathfrak{a} m}^{\prime}(z, s, 1, k ; \bar{f})=\left(s+\frac{k}{2}\right) Q_{\mathfrak{a} m}(z, s, 1, k+2 ; \bar{f})- \\
& 4 \pi m Q_{\mathfrak{a} m}(z, s+1,1, k+2 ; \bar{f})-\frac{k}{2} Q_{\mathfrak{a} m}(z, s, 1, k ; \bar{f}) \\
& 7
\end{aligned}
$$


This together with the continuation and bounds of $Q_{\mathfrak{a} m}$ that we have just proved yields the desired result about $Q_{\mathfrak{a} m}^{\prime}$.

\subsection{The basic theorem}

We are ready to state the theorem that will enable us to construct the basis elements for $S_{2}^{t}$.

We first construct a family of elementary functions in $S_{2}^{t}$. Fix a cusp a and let $\left\{f_{1}, \ldots, f_{g}\right\}$ be an orthonormal basis of $S_{2}$. For $i_{j} \in\{1, \ldots, g\}$ we set

$$
F_{i_{1}, \ldots, i_{t}}(z)=f_{i_{1}}(z) \int_{\mathfrak{a}}^{z} f_{i_{2}}(w)\left(\int_{\mathfrak{a}}^{w} f_{i_{3}} \ldots\right) d w .
$$

It is easy to see with (2.3) and an inductive argument that

$$
\left.F_{i_{1}, \ldots, i_{t}}\right|_{2}(\gamma-1)=\sum_{r=1}^{t-1} F_{i_{1}, \ldots, i_{r}} \int_{\mathfrak{a}}^{\gamma \mathfrak{a}} F_{i_{r+1}, \ldots, i_{t}}(w) d w
$$

It is straightforward, by an inductive argument, to see that they are invariant under the parabolic elements, that they vanish at the cusps and that $\left.F_{i_{1}, \ldots, i_{t}}\right|_{2}(\gamma-1) \in S_{2}^{t-1}$. Hence $F_{i_{1}, \ldots, i_{t}} \in S_{2}^{t}$.

Since these functions will play a fundamental role in the sequel, we set

$$
A_{t}=\left\{F_{i_{1}, \ldots, i_{t}} ; i_{j} \in\{1, \ldots, g\}\right\} .
$$

It is clear that, if $f \in A_{t},\left.f\right|_{2}(\cdot-1) \in \mathcal{H}_{t}$, so the results of the previous sections apply to the elements of this set.

To generate further higher-order cusp forms, we need some functions that depend on standard cusp forms in a less elementary way than the $F_{i_{1}, \ldots, i_{t}}$ 's do. Specifically, let $m \geq 0$ and $k \in 2 \mathbb{Z}$. For $f \in S_{2}^{t}(\Gamma)$ we set

$$
Z_{\mathfrak{a} m}(z, s, 1, k ; \bar{f}):=\sum_{\gamma \in \Gamma_{\mathfrak{a}} \backslash \Gamma} \overline{\left(\int_{\mathfrak{a}}^{\gamma \mathfrak{a}} f(w) d w\right)} \operatorname{Im}\left(\sigma_{\mathfrak{a}}{ }^{-1} \gamma z\right)^{s} e\left(m \sigma_{\mathfrak{a}}{ }^{-1} \gamma z\right) \varepsilon\left(\sigma_{\mathfrak{a}}{ }^{-1} \gamma, z\right)^{-k}
$$

This function is essentially a generalization of the function $Z_{\mathfrak{a} m}(z, s ; f)$ which was crucial for the construction of a basis of the space of second-order cusp forms in [DO]. However, the multiplier is slightly modified. The advantage is that in this way we avoid the introduction of the function $G_{\mathfrak{a} m}$ used in [DO].

We need to meromorphically continue $Z_{\mathfrak{a} m}$ to a region that contains 1 . The proof has many similarities to that of the corresponding result in [DO].

Theorem 3.4. For $f \in A_{t}, Z_{\mathfrak{a} m}(z, s, 1, k ; \bar{f})$ admits a meromorphic continuation to $\operatorname{Re}(s)>1-\delta_{\Gamma}$. The only possible pole is $s=1$ and it can only occur when $k \leq 0$. For $k=0$, it is simple. For $k \geq 2$, and $m \neq 0, Z_{\mathfrak{a} m}(z, s, 1, k ; \bar{f}) \ll y_{\mathfrak{F}}(z)^{1 / 2}$. For $k \geq 2, Z_{\mathfrak{a} 0}(z, s, 1, k ; \bar{f}) \ll y_{\mathfrak{F}}(z)^{\sigma}$. For $k=2$, $Z_{\mathfrak{a} 0}(z, 1,1,2 ; \bar{f}) \ll y_{\mathfrak{F}}(z)^{1 / 2}$. The implied constants are independent of $z$ in all cases.

Proof: We will prove the theorem by induction on $t$. For $t=0$, it is trivial. Let $t>0$ and suppose, the statement is true for orders $<t$. If $f \in A_{t}$, then (2.3) implies

$$
\begin{aligned}
Z_{\mathfrak{a} m}(z, s, 1, k ; \bar{f})=Q_{\mathfrak{a} m}(z, s, 1, k ; \bar{f})-\overline{\left(\int_{\mathfrak{a}}^{z} f(w) d w\right)} & U_{\mathfrak{a} m}(z, s, k)+ \\
& \sum^{\prime} Z_{\mathfrak{a} m}(z, s, 1, k ; \bar{h})\left(\overline{\left.\int_{z}^{\mathfrak{a}} h_{1}(w) d w\right)}\right.
\end{aligned}
$$

where the prime indicates that the summation is over some pairs $\left(h, h_{1}\right) \in A_{r} \times A_{t-r}, 1 \leq r \leq t-1$.

The meromorphic continuation and bounds of $U_{\mathfrak{a} m}(z, s, k)$ and $Z_{\mathfrak{a} m}(z, s, 1, k ; \bar{h})$ in $(3.14)$ are known by Prop. 3.2 and the inductive hypothesis respectively. Therefore, we only need to meromorphically continue and bound $Q_{\mathfrak{a} m}(z, s, 1, k ; \bar{f})$. To this end we first need to study $Q_{\mathfrak{a} m}(z, s+n+1,-n, k, \bar{f})$ for $n \geq 0$. 
Proposition 3.5. Suppose that $f \in A^{t}$. For $k \in \mathbb{Z}$ and $-n \leqslant 0$ the series $Q_{\mathfrak{a} m}(z, s+n+1,-n, k, \bar{f})$ has a meromorphic continuation to $\operatorname{Re}(s)>1-\delta_{\Gamma}$. For $k \geq 0$, it is analytic. Also for these $s$ and $k \geq 0$ we have $Q_{\mathfrak{a} m}(z, s+n+1,-n, k ; \bar{f}) \ll e^{-\pi y_{\Gamma}(z)}$ with the implied constant depending on $n, m, f, k, s$ and $\Gamma$ alone.

Proof: We begin with the formula

$$
\overline{g^{(n)}(\gamma z)}=(-2 i)^{-n} \operatorname{Im}(\gamma z)^{-n-1} \sum_{r=0}^{n}(-1)^{n-r} \varepsilon(\gamma, z)^{-2 r-2}\left(\begin{array}{c}
n \\
r
\end{array}\right) \frac{(n+1) !}{(r+1) !} L^{r}\left(\theta_{\gamma,-2}(y \overline{g(z)})\right)
$$

valid for any holomorphic $g: \mathfrak{H} \rightarrow \mathbb{C}$ and for all $\gamma$ in $\Gamma$ (see [CO] for a proof). Set $\Gamma^{\prime}:=\sigma_{\mathfrak{a}}{ }^{-1} \Gamma \sigma_{\mathfrak{a}}$. Then $\sigma_{\mathfrak{a}}{ }^{-1} \Gamma_{\mathfrak{a}} \sigma_{\mathfrak{a}}=\Gamma_{\infty}$ and

$$
\begin{gathered}
\varepsilon\left(\sigma_{\mathfrak{a}}, z\right)^{-k} Q_{\mathfrak{a} m}\left(\sigma_{\mathfrak{a}} z, s,-n, k ; \bar{f}\right)=\sum_{\gamma^{\prime} \in \Gamma_{\infty} \backslash \Gamma^{\prime}} \overline{f_{\mathfrak{a}}^{(n)}\left(\gamma^{\prime} z\right)} \operatorname{Im}\left(\gamma^{\prime} z\right)^{s} e\left(m \gamma^{\prime} z\right) \varepsilon\left(\gamma^{\prime}, z\right)^{-k}= \\
(-2 i)^{-n} \sum_{r=0}^{n}(-1)^{n-r}\left(\begin{array}{l}
n \\
r
\end{array}\right) \frac{(n+1) !}{(r+1) !} \sum_{\gamma^{\prime} \in \Gamma_{\infty} \backslash \Gamma^{\prime}} L^{r}\left(\theta_{\gamma^{\prime},-2}\left(y \overline{f_{\mathfrak{a}}(z)}\right)\right) \operatorname{Im}\left(\gamma^{\prime} z\right)^{s-n-1} e\left(m \gamma^{\prime} z\right) \varepsilon\left(\gamma^{\prime}, z\right)^{-(2 r+2)-k}
\end{gathered}
$$

Now, if $\left.f\right|_{2}(\gamma-1)=\sum^{\prime}\left(\int_{\mathfrak{a}}^{\gamma \mathfrak{a}} h(w) d w\right) h_{1}(\gamma \in \Gamma)$, with $\left(h, h_{1}\right) \in A_{r}(\Gamma) \times A_{t-r}(\Gamma),(r \geq 1)$, then $\left.f_{\mathfrak{a}}\right|_{2}\left(\gamma^{\prime}-1\right)=\sum^{\prime}\left(\int_{\sigma_{\mathfrak{a}}-1}^{\gamma^{\prime} \sigma_{\mathfrak{a}}}{ }^{-1} \mathfrak{a} h_{\mathfrak{a}}(w) d w\right)\left(h_{1}\right)_{\mathfrak{a}}$ for $\gamma^{\prime} \in \Gamma^{\prime}$ and $\left(h_{\mathfrak{a}},\left(h_{1}\right)_{\mathfrak{a}}\right) \in A_{r}\left(\Gamma^{\prime}\right) \times A_{t-r}\left(\Gamma^{\prime}\right)$. Therefore,

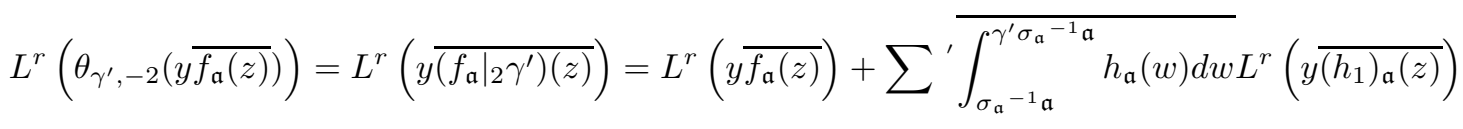

It is also easy to see (cf. Prop. E of $[\mathrm{DO}]$ ) that for all $G: \mathfrak{H} \rightarrow \mathbb{C}$,

$$
L^{r}\left(y \overline{G_{\mathfrak{a}}(z)}\right)=\left.L^{r}(y \overline{G(z)})\right|_{\sigma_{\mathfrak{a}} z} \varepsilon\left(\sigma_{\mathfrak{a}}, z\right)^{2 r+2}
$$

so

$$
\begin{aligned}
& \sum_{\gamma^{\prime} \in \Gamma_{\infty} \backslash \Gamma^{\prime}} L^{r}\left(\theta_{\gamma^{\prime},-2}\left(y \overline{f_{\mathfrak{a}}(z)}\right)\right) \operatorname{Im}\left(\gamma^{\prime} z\right)^{s-n-1} e\left(m \gamma^{\prime} z\right) \varepsilon\left(\gamma^{\prime}, z\right)^{-(2 r+2)-k}= \\
& \left.L^{r}(\overline{f(z)})\right|_{\sigma_{\mathfrak{a}} z} U_{\mathfrak{a} m}\left(\sigma_{\mathfrak{a}} z, s-n-1,2 r+2+k\right) \varepsilon\left(\sigma_{\mathfrak{a}}, z\right)^{-k}+ \\
& \sum_{\gamma \in \Gamma_{\mathfrak{a}} \backslash \Gamma}^{\prime}\left[L^{r}\left(y \overline{h_{1}(z)}\right) \times\right. \\
& =L^{r}\left(\left.\overline{\left.\int_{\mathfrak{a}}^{\gamma \mathfrak{a}} h(z)\right)}\right|_{\sigma_{\mathfrak{a}} z} U_{\mathfrak{a} m}\left(\sigma_{\mathfrak{a}} z, s-n-1,2 r+2+k\right) \varepsilon\left(\sigma_{\mathfrak{a}}, z\right)^{-k}+\right. \\
& \left.\sum^{\prime} L^{r}\left(y \overline{h_{1}(z)}\right)\right|_{\sigma_{\mathfrak{a}} z} Z_{\mathfrak{a} m}\left(\sigma_{\mathfrak{a}} z, s-n-1,1,2 r+2+k ; \bar{h}\right) \varepsilon\left(\sigma_{\mathfrak{a}}, z\right)^{-k}
\end{aligned}
$$

Since $h \in A_{r}, r<t$, the inductive hypothesis implies that $Z_{\mathfrak{a} m}$ has a meromorphic continuation for $\operatorname{Re}(s-n-1)>1-\delta_{\Gamma}$. By Prop. 3.2 the same holds for $U_{\mathfrak{a} m}$. Moreover, since $2 r+2 \geq 2$, by the inductive hypothesis and Prop. 3.2 we deduce that we obtain an analytic function when $k \geq 0$.

The function $L^{r}(y \overline{f(z)})$ (and $L^{r}(y \overline{h(z)})$ ) has exponential decay at every cusp $\mathfrak{b}$ because

$$
\theta_{\sigma_{\mathfrak{b}},-2 r-2} L^{r}(y \overline{f(z)})=L^{r}\left(\theta_{\sigma_{\mathfrak{b}},-2} y \overline{f(z)}\right)=L^{r}\left(y \overline{j\left(\sigma_{\mathfrak{b}} z\right)^{-2} f\left(\sigma_{\mathfrak{b}} z\right)}\right)=L^{r}\left(y \sum_{n=1}^{\infty} \overline{a_{\mathfrak{b}}(n) e(n z)}\right) .
$$


Hence

$$
L^{r}(y \overline{f(z)}) \ll y_{\Gamma}(z)^{r+1} e^{-2 \pi y_{\Gamma}(z)}
$$

for an implied constant depending on $r, f$ and $\Gamma$. Therefore, with (3.15), (3.16), Prop. 3.2 and the inductive hypothesis, we have for $\operatorname{Re}(s)>1-\delta_{\Gamma}$ and $k \geq 0$ :

$$
Q_{\mathfrak{a} m}(z, s+n+1,-n, k ; \bar{f}) \ll e^{-\pi y_{\Gamma}(z)} .
$$

We are now ready to prove the analytic continuation of $Q_{\mathfrak{a} m}(z, s, 1, k, \bar{f})$.

Proposition 3.6. Let $m \geq 0$ and $k \in 2 \mathbb{Z}$. For $f \in A_{t}$ the series $Q_{\mathfrak{a} m}(z, s, 1, k ; \bar{f})$ has continuation to a meromorphic function of $s$ with $\operatorname{Re}(s)>1-\delta_{\Gamma}$. For $k>0$, we obtain an analytic function. For $k=0$, it has only a simple pole at $s=1$ with residue $\frac{-\overline{a_{\mathfrak{a}}(m)}}{2 \pi i m}$ if $m \neq 0$ and 0 otherwise. For $k \neq 0$ or $k=m=0$, we have $Q_{\mathfrak{a} m}(z, s, 1, k ; \bar{f}) \ll y_{\Gamma}(z)^{1 / 2}$. For $k=0, m \neq 0,(s-1) Q_{\mathfrak{a} m}(z, s, 1,0 ; \bar{f}) \ll$ $y_{\Gamma}(z)^{1 / 2}$. The implied constants depend on $s, m, f$ and $\Gamma$.

Proof: We first prove the result for $k=0$. By Proposition $3.3, Q_{\mathfrak{a} m}(z, s, 1,0 ; \bar{f})$ is square integrable for $\operatorname{Re}(s)>1$ and the spectral decomposition yields

$$
\begin{aligned}
Q_{\mathfrak{a} m}(z, s, 1,0 ; \bar{f})=\sum_{j=0}^{\infty}\left\langle Q_{\mathfrak{a} m}(\cdot, s, 1,0 ; \bar{f}), \eta_{j}\right\rangle \eta_{j} \\
\quad+\frac{1}{4 \pi} \sum_{\mathfrak{b}} \int_{-\infty}^{\infty}\left\langle Q_{\mathfrak{a} m}(\cdot, s, 1,0 ; \bar{f}), E_{\mathfrak{b}}(\cdot, 1 / 2+i r)\right\rangle E_{\mathfrak{b}}(z, 1 / 2+i r) d r .
\end{aligned}
$$

We recall the Prop. 9.3 and Cor. 9.4 of [JO]:

Lemma 3.7. Let $\xi_{1}, \xi_{2}$ and $\psi$ be any smooth $\Gamma$ invariant functions (not necessarily in $L^{2}(\Gamma \backslash \mathfrak{H})$ ). If $(\Delta-\lambda) \xi_{1}=\xi_{2},\left(\Delta-\lambda^{\prime}\right) \psi=0$ and

$$
\begin{aligned}
\xi_{1}, R_{0} \xi_{1}, \Delta \xi_{1} & \ll y_{\Gamma}(z)^{A}, \\
\psi, R_{0} \psi & \ll y_{\Gamma}(z)^{B}
\end{aligned}
$$

for $A+B<0$ and $R_{0}=2 i y \frac{d}{d z}$ the raising operator, then

$$
\left\langle\xi_{1}, \psi\right\rangle=\frac{1}{\lambda^{\prime}-\lambda}\left\langle\xi_{2}, \psi\right\rangle .
$$

We will apply this lemma to $\xi_{1}=Q_{\mathfrak{a} m}(z, s, n, 0 ; \bar{f})(n \in \mathbb{Z})$ and $\psi=\eta_{j}$. (3.1) implies that for all $n \in \mathbb{Z}$

$$
\begin{aligned}
(\Delta-s(1-s)) Q_{\mathfrak{a} m}(z, s, n, 0 ; \bar{f})= & -8 \pi i m Q_{\mathfrak{a} m}(z, s+2, n-1,0 ; \bar{f}) \\
& +4 \pi m s Q_{\mathfrak{a} m}(z, s+1, n, 0 ; \bar{f})+2 i s Q_{\mathfrak{a} m}(z, s+1, n-1,0 ; \bar{f}) .
\end{aligned}
$$

Next, we have $\eta_{j}(z), R_{0} \eta_{j}(z) \ll y_{\Gamma}(z)^{1 / 2}$ by Lemma 3.1(i) and

$$
Q_{\mathfrak{a} m}(z, s, 1,0 ; \bar{f}), R_{0} Q_{\mathfrak{a} m}(z, s, 1,0 ; \bar{f}), \Delta Q_{\mathfrak{a} m}(z, s, 1,0 ; \bar{f}) \ll y_{\Gamma}(z)^{1 / 2-\sigma / 2}
$$

for $\sigma=\operatorname{Re}(s)>1$ by Proposition 3.3 and Proposition 3.5. So we may use Lemma 3.7 to get, for $\operatorname{Re}(s)>2$,

$$
\begin{aligned}
\left\langle Q_{\mathfrak{a} m}(\cdot, s, 1,0 ; \bar{f}), \eta_{j}\right\rangle=\frac{1}{\left(s_{j}-s\right)\left(1-s_{j}-s\right)}\left(-8 \pi i m\left\langle Q_{\mathfrak{a} m}(\cdot, s+2,0,0 ; \bar{f}), \eta_{j}\right\rangle\right. \\
\left.+4 \pi m s\left\langle Q_{\mathfrak{a} m}(\cdot, s+1,1,0 ; \bar{f}), \eta_{j}\right\rangle+2 i s\left\langle Q_{\mathfrak{a} m}(\cdot, s+1,0,0 ; \bar{f}), \eta_{j}\right\rangle\right) .
\end{aligned}
$$


We can repeat this procedure $W$ times in all to obtain, again for $\operatorname{Re}(s)>2$,

$$
\left\langle Q_{\mathfrak{a} m}(\cdot, s, 1,0 ; \bar{f}), \eta_{j}\right\rangle=\sum_{l} \frac{P_{l}(m, s)}{R_{l}\left(s_{j}, s\right)}\left\langle Q_{\mathfrak{a} m}\left(\cdot, s+W+c_{l}, 1-d_{l}, 0 ; \bar{f}\right), \eta_{j}\right\rangle,
$$

with integers $c_{l}, d_{l}$ satisfying $0 \leqslant c_{l}, d_{l} \leqslant W, d_{l} \leq W+c_{l}, P_{l}(m, s)$ a polynomial in $m, s$ alone of degree $W$ in $m$ and of degree $W$ in $s$ and $R_{l}\left(s_{j}, s\right)$ a polynomial in $s_{j}, s$ alone of degree $2 W$ in $s_{j}$ and of degree $2 W$ in $s$. In fact

$$
R_{l}\left(s_{j}, s\right)=\prod_{b}\left(s_{j}-b-s\right)\left(1-s_{j}-b-s\right)
$$

where, for each $l$, the product is over some subset of integers $b$ in $\{0,1, \cdots, 2 W\}$ of cardinality $W$.

If $d_{l}=0$, then we have

$$
Q_{\mathfrak{a} m}\left(z, s+W+c_{l}, 1,0 ; \bar{f}\right) \ll y_{\Gamma}(z)^{1 / 4-W / 2}
$$

by Proposition 3.3, for $W \geqslant 1$. Hence

$$
\left\langle Q_{\mathfrak{a} m}\left(\cdot, s+W+c_{l}, 1,0 ; \bar{f}\right), \eta_{j}\right\rangle \ll \sqrt{\left\|y_{\Gamma}(z)^{-1 / 4}\right\| \cdot\left\|\eta_{j}\right\|}=\sqrt{\left\|y_{\Gamma}(z)^{-1 / 4}\right\|} \ll 1 .
$$

For $0<d_{l} \leqslant W$, Prop. 3.5 implies that

$$
Q_{\mathfrak{a} m}\left(z, s+W+c_{l}, 1-d_{l}, 0 ; \bar{f}\right) \ll e^{-\pi y_{\Gamma}(z)}
$$

and hence $\left\langle Q_{\mathfrak{a} m}\left(z, s+W+c_{l}, 1-d_{l}, 0 ; \bar{f}\right), \eta_{j}\right\rangle \ll 1$. Therefore, for $j>0,\left\langle Q_{\mathfrak{a} m}(\cdot, s, 1,0 ; \bar{f}), \eta_{j}\right\rangle$ is an analytic function of $s$ for $\operatorname{Re}(s)>1-\delta_{\Gamma}$ and satisfies

$$
\left\langle Q_{\mathfrak{a} m}(\cdot, s, 1,0 ; \bar{f}), \eta_{j}\right\rangle \ll\left|s_{j}\right|^{-2 W} \ll\left|\lambda_{j}\right|^{-W}
$$

for implied constants depending on $s, m, W, f$ and $\Gamma$ alone and with the dependence on $s$ being uniform on compacta.

For $j>0$ and for all $n \geq 0$ we can now use (3.4), Lemma 3.1 (i) and (3.22) to get

$$
\sum_{T \leqslant\left|\lambda_{j}\right|<T+1}<Q_{\mathfrak{a} m}(\cdot, s, 1,0 ; \bar{f}), \eta_{j}>R^{n}\left(\eta_{j}(z)\right) \ll T^{1-W}\left(\left(T^{n / 2}+1\right) y_{\Gamma}(z)^{1 / 2}+\left(T^{n+7 / 2}+1\right) y_{\Gamma}(z)^{-3 / 2}\right) .
$$

Hence for $W=6+n$,

$$
\sum_{j=1}^{\infty}<Q_{\mathfrak{a} m}(\cdot, s, 1,0 ; \bar{f}), \eta_{j}>R^{n}\left(\eta_{j}(z)\right) \ll y_{\Gamma}(z)^{1 / 2}
$$

for all $s$ with $\operatorname{Re}(s)>1-\delta_{\Gamma}$. The sum converges uniformly for $s$ in compact sets with $\operatorname{Re}(s)>1-\delta_{\Gamma}$ giving an analytic function of $s$. When $n>0, R^{n}$ eliminates $\left\langle Q_{\mathfrak{a} m}(\cdot, s, 1,0 ; \bar{f}), \eta_{0}\right\rangle \eta_{0}(z)$.

For $j=0$, the constant eigenfunction is $\eta_{0}=V^{-1 / 2}$. If $m \neq 0$, by unfolding we obtain

$$
\left\langle Q_{\mathfrak{a} m}(\cdot, s, 1,0 ; \bar{f}), \eta_{0}\right\rangle \eta_{0}=\frac{-\overline{a_{\mathfrak{a}}(m)} \Gamma(s-1)}{2 \pi i m(4 \pi m)^{s-1}}=\frac{-\overline{a_{\mathfrak{a}}(m)}}{2 \pi i m}\left(\frac{1}{s-1}+O(1)\right)
$$

as $s \rightarrow 1$ for $f_{\mathfrak{a}}(z)=\sum_{m=1}^{\infty} a_{\mathfrak{a}}(m) e(m z)$. If $m=0$, the same process gives 0 . 
With arguments similar to those used for the discrete spectrum we now consider the continuous spectrum. For $P_{l}, R_{l}, c_{l}$ and $d_{l}$ identical to (3.18), Lemma 3.1 (ii) gives

$$
\begin{aligned}
& \left\langle Q_{\mathfrak{a} m}(\cdot, s, 1,0 ; \bar{f}), E_{\mathfrak{b}}(\cdot, 1 / 2+i r)\right\rangle= \\
& \quad \sum_{l} \frac{P_{l}(m, s)}{R_{l}(1 / 2+i r, s)}\left\langle Q_{\mathfrak{a} m}\left(\cdot, s+W+c_{l}, 1-d_{l}, 0 ; \bar{f}\right), E_{\mathfrak{b}}(\cdot, 1 / 2+i r)\right\rangle,
\end{aligned}
$$

which is true for $\operatorname{Re}(s)>2$ initially.

With (3.19), (3.20), Prop. 3.5 and Lemma 3.1 (ii) we see that (for $W \geqslant 1$ ) the right side of (3.24) converges and gives the analytic continuation of the left side to $\operatorname{Re}(s)>1-\delta_{\Gamma}$. Now, for all $n \geq 0$,

$$
\begin{aligned}
& \int_{T}^{T+1}\left\langle Q_{\mathfrak{a} m}(\cdot, s, 1,0 ; \bar{f}), E_{\mathfrak{b}}(\cdot, 1 / 2+i r)\right\rangle R^{n} E_{\mathfrak{b}}\left(z_{0}, 1 / 2+i r\right) d r \\
& \quad=\sum_{l} P_{l}(m, s) \int_{T}^{T+1} \frac{\left\langle Q_{\mathfrak{a} m}\left(\cdot, s+W+c_{l}, 1-d_{l}, 0 ; \bar{f}\right), E_{\mathfrak{b}}(\cdot, 1 / 2+i r)\right\rangle}{R_{l}(1 / 2+i r, s)} R^{n} E_{\mathfrak{b}}\left(z_{0}, 1 / 2+i r\right) d r \\
& \quad=\sum_{l} P_{l}(m, s) \int_{T}^{T+1} \int_{\mathfrak{F}} \frac{Q_{\mathfrak{a} m}\left(z, s+W+c_{l}, 1-d_{l}, 0 ; \bar{f}\right)}{R_{l}(1 / 2+i r, s)} \overline{E_{\mathfrak{b}}(z, 1 / 2+i r)} R^{n} E_{\mathfrak{b}}\left(z_{0}, 1 / 2+i r\right) d \mu z d r .
\end{aligned}
$$

The integrand satisfies

$$
\begin{aligned}
\frac{Q_{\mathfrak{a} m}\left(z, s+W+c_{l}, 1-d_{l}, 0 ; \bar{f}\right)}{R_{l}(1 / 2+i r, s)} \overline{E_{\mathfrak{b}}(z, 1 / 2+i r)} R^{n} E_{\mathfrak{b}}\left(z_{0}, 1 / 2+i r\right) & \ll \\
& |r|^{-2 W+n} y_{\Gamma}(z)^{1 / 4-W / 2} y_{\Gamma}(z)^{1 / 2} y_{\Gamma}\left(z_{0}\right)^{1 / 2}
\end{aligned}
$$

by (3.19), (3.20), Prop. 3.5, Lemma 3.1(ii) and the easily proved identity

$$
R^{n} E_{\mathfrak{a}}(z, s)=s(s+1) \cdots(s+n-1) U_{\mathfrak{a} 0}(z, s, 2 n) .
$$

Thus the double integral in (3.25) is absolutely and uniformly convergent and we may interchange the limits of integration to obtain

$$
\sum_{l} P_{l}(m, s) \int_{\mathfrak{F}} Q_{\mathfrak{a} m}\left(z, s+W+c_{l}, 1-d_{l}, 0 ; \bar{f}\right) \int_{T}^{T+1} \frac{\overline{E_{\mathfrak{b}}(z, 1 / 2+i r)}}{R_{l}(1 / 2+i r, s)} R^{n} E_{\mathfrak{b}}\left(z_{0}, 1 / 2+i r\right) d r d \mu z
$$

Also

$$
\begin{aligned}
\int_{T}^{T+1} \frac{\overline{E_{\mathfrak{b}}(z, 1 / 2+i r)}}{R_{l}(1 / 2+i r, s)} & R^{n} E_{\mathfrak{b}}\left(z_{0}, 1 / 2+i r\right) d r \\
& \ll T^{-2 W} \sqrt{\int_{T}^{T+1}\left|E_{\mathfrak{b}}(z, 1 / 2+i r)\right|^{2} d r \cdot \int_{T}^{T+1}\left|R^{n} E_{\mathfrak{b}}\left(z_{0}, 1 / 2+i r\right)\right|^{2} d r}
\end{aligned}
$$

So, with Lemma 3.1 (iii), (3.26) is bounded by a constant times

$$
\sum_{l}\left|P_{l}(m, s)\right| T^{12-2 W+2 n} \int_{\mathfrak{F}} y_{\Gamma}(z)^{3 / 4-W / 2} d \mu z \cdot y_{\Gamma}\left(z_{0}\right)^{1 / 2} .
$$

This means that, for $W$ chosen large enough,

$$
\int_{-\infty}^{\infty}\left\langle Q_{\mathfrak{a} m}(\cdot, s, 1,0 ; \bar{f}), E_{\mathfrak{b}}(\cdot, 1 / 2+i r)\right\rangle R^{n} E_{\mathfrak{b}}(z, 1 / 2+i r) d r \ll y_{\Gamma}(z)^{1 / 2} .
$$


To combine the information we have collected for the discrete and the continuous part of (3.17) we observe that with (3.23) and preceeding discussion we can interchange summation and differentation to get

$$
R^{n}\left(\sum_{j=1}^{\infty}<Q_{\mathfrak{a} m}(\cdot, s, 1,0 ; \bar{f}), \eta_{j}>\eta_{j}(z)\right)=\sum_{j=1}^{\infty}<Q_{\mathfrak{a} m}(\cdot, s, 1,0 ; \bar{f}), \eta_{j}>R^{n}\left(\eta_{j}(z)\right)
$$

and that Lemma 10.2 of [DO] implies

$$
\begin{aligned}
\frac{1}{4 \pi} \sum_{\mathfrak{b}} R^{n} \int_{T}^{T+1}\left\langle Q_{\mathfrak{a} m}(\cdot, s, 1,0 ; \bar{f}), E_{\mathfrak{b}}(\cdot, 1 / 2+i r)\right\rangle E_{\mathfrak{b}}(z, 1 / 2+i r) d r= \\
\frac{1}{4 \pi} \sum_{\mathfrak{b}} \int_{T}^{T+1}\left\langle Q_{\mathfrak{a} m}(\cdot, s, 1,0 ; \bar{f}), E_{\mathfrak{b}}(\cdot, 1 / 2+i r)\right\rangle R^{n} E_{\mathfrak{b}}(z, 1 / 2+i r) d r
\end{aligned}
$$

for all $T \in \mathbb{R}$. Therefore,

$$
\begin{aligned}
R^{n} Q_{\mathfrak{a} m}(z, s, 1,0 ; \bar{f})=\left\langle Q_{\mathfrak{a} m}(\cdot, s, 1,0 ; \bar{f}), \eta_{0}\right\rangle R^{n} \eta_{0}+\sum_{j=1}^{\infty}\left\langle Q_{\mathfrak{a} m}(\cdot, s, 1,0 ; \bar{f}), \eta_{j}\right\rangle R^{n}\left(\eta_{j}(z)\right) \\
\quad+\frac{1}{4 \pi} \sum_{\mathfrak{b}} \int_{-\infty}^{\infty}\left\langle Q_{\mathfrak{a} m}(\cdot, s, 1,0 ; \bar{f}), E_{\mathfrak{b}}(\cdot, 1 / 2+i r)\right\rangle R^{n} E_{\mathfrak{b}}(z, 1 / 2+i r) d r
\end{aligned}
$$

and it has a continuation to a meromorphic function of $s$ with $\operatorname{Re}(s)>1-\delta_{\Gamma}$. For these values, $(s-1) Q_{\mathfrak{a} m}(z, s, 1,0 ; \bar{f}), R^{n} Q_{\mathfrak{a} m}(z, s, 1,0 ; \bar{f})(n>0)$ and $Q_{\mathfrak{a} 0}(z, s, 1,0 ; \bar{f})$ are all $\ll y_{\Gamma}(z)^{1 / 2}$ The only pole is at $s=1$ and it comes from the contribution of $\eta_{0}$ when $n=0$.

To pass to general $k$ 's we apply the operators $R_{r}$ successively, using (3.1), to obtain, for $k>0$ :

$$
\begin{aligned}
Q_{\mathfrak{a} m}(z, s, 1, k ; \bar{f})=\frac{1}{s(s+1) \cdots\left(s+\frac{k}{2}-1\right)} & R^{k} Q_{\mathfrak{a} m}(z, s, 1,0 ; \bar{f}) \\
& \left.+\sum_{i=-1}^{k-1} \sum_{j=1+i}^{k}{ }^{\prime} p_{i, j}(m, s) Q_{\mathfrak{a} m}(z, s+j,-i, k ; \bar{f})\right)
\end{aligned}
$$

with polynomials $p_{i, j}$ in $m$ and $s$. Here the prime indicates that we exclude the term corresponding to $(i, j)=(-1,0)$. Thanks to Propositions $3.3,3.5$ (for $-i \leq 0)$ and the meromorphic continuation and growth of $R^{k} Q_{\mathfrak{a} m}(z, s, 1,0 ; f)$ we just proved, the identity (3.27) implies Prop. 3.6. For $k<0$, we work in a similar way.

End of proof of Th. 3.4 By Propositions 3.2 and 3.6, the inductive hypothesis and (3.14), we deduce that for $k>0 Z_{\mathfrak{a} m}(z, s, 1, k ; \bar{f})$ is holomorphic in $s$ and that for $k=0$ the only possible pole is at $s=1$ which is simple. This completes the proof of the analytic continuation of $Z_{\mathfrak{a} m}$.

To prove the bounds, we recall from (3.11) and (3.12) that, for $z \in \mathfrak{F}, \int_{\mathfrak{a}}^{\sigma_{\mathfrak{a}} z} f(w) d w \ll 1$ and $\int_{\mathfrak{a}}^{\sigma_{\mathfrak{b}} z} f(w) d w \ll e^{-2 \pi y}$, if $\mathfrak{a} \neq \mathfrak{b}$ as $y \rightarrow \infty$. The desired bound follows from this, Propositions 3.2 and 3.6, the inductive hypothesis and (3.14). For $m=0, k=2$ and $s=1$ we deduce the bound from these inequalities and $U_{\mathfrak{a} 0}\left(\sigma_{\mathfrak{b}} z, 1,2\right) \ll \delta_{\mathfrak{a} \mathfrak{b}} y+1$, as $y \rightarrow \infty$ (see $\left.(3.8)\right)$.

\subsection{A family of functions of $S_{2}^{t}$.}

In this section we construct a family of $t$-order cusp forms based on the analytic continuation of $Z_{\mathfrak{a} m}(z, s, 1,2 ; f)$ established in Section 3.2. The construction is carried out in three steps.

In the first step, since we are mainly interested in the weight according to $j(\gamma, z)$ rather than $\varepsilon(\gamma, z)$, we set

$$
\begin{aligned}
Z_{\mathfrak{a} m}(z, s ; \bar{f}) & :=y^{-1} Z_{\mathfrak{a} m}(z, s+1,1,2 ; \bar{f}) \\
& =\sum_{\gamma \in \Gamma_{\mathfrak{a}} \backslash \Gamma} \overline{\left(\int_{\mathfrak{a}}^{\gamma \mathfrak{a}} f(w) d w\right)} \operatorname{Im}\left(\sigma_{\mathfrak{a}}{ }^{-1} \gamma z\right)^{s} e\left(m \sigma_{\mathfrak{a}}{ }^{-1} \gamma z\right) j\left(\sigma_{\mathfrak{a}}{ }^{-1} \gamma, z\right)^{-2} .
\end{aligned}
$$


According to Theorem $3.4, Z_{\mathfrak{a} m}\left(z, s, \bar{F}_{i_{1}, \ldots, i_{t-1}}\right)$ is analytic for $\operatorname{Re}(s)>-\delta_{\Gamma}$. It is easy to see that

$$
\left.Z_{\mathfrak{a} m}\left(\cdot, 0, \bar{F}_{i_{1}, \ldots, i_{t-1}}\right)\right|_{2}(\gamma-1)=\overline{\left(\int_{\mathfrak{a}}^{\gamma^{-1} \mathfrak{a}} F_{i_{1}, \ldots, i_{t-1}}\right)} P_{\mathfrak{a} m}+\sum_{r=1}^{t-2} \overline{\left(\int_{\mathfrak{a}}^{\gamma^{-1} \mathfrak{a}} F_{i_{1}, \ldots, i_{r}}\right)} Z_{\mathfrak{a} m}\left(\cdot, 0, \bar{F}_{i_{r+1}, \ldots, i_{t-1}}\right) .
$$

Further, for $\operatorname{Re}(s)$ large we have:

$$
\frac{d}{d \bar{z}} Z_{\mathfrak{a} m}\left(z, s ; \bar{F}_{i_{1}, \ldots, i_{t-1}}\right)=\frac{i s}{2 y^{2}} Z_{\mathfrak{a} m}\left(z, s+1,1,0 ; \bar{F}_{i_{1}, \ldots, i_{t-1}}\right)
$$

In the second step, if $f_{i_{t}}=\sum_{l} a_{l} P_{\mathfrak{a} m_{l}}$, we set, for $j \geq 1$,

$$
Z_{i_{j}, \ldots, i_{t}}=\sum_{l} a_{l} Z_{\mathfrak{a} m_{l}}\left(\cdot, 0 ; \bar{F}_{i_{j}, \ldots, i_{t-1}}\right) .
$$

An inductive argument implies that, for $t \geq 2$,

$$
\left.Z_{i_{1}, \ldots, i_{t}}\right|_{2}(\gamma-1)=\sum_{r=1}^{t-1} \overline{\left(\overline{\int_{\mathfrak{a}}^{\gamma^{-1} \mathfrak{a}} F_{i_{1}, \ldots, i_{r}}}\right)} Z_{i_{r+1}, \ldots, i_{t}}
$$

In view of (3.29), we apply the same linear combination to $\operatorname{Res}_{s=1} Z_{\mathfrak{a} m}\left(\cdot, s, 1,0 ; \bar{F}_{i_{1}, \ldots, i_{t-1}}\right)$ and to $\frac{-\overline{a_{a}(m)}}{2 \pi i m}$, for $m>0$. We denote them by $R_{i_{1}, \ldots, i_{t}}$ and $a_{i_{1}, \ldots, i_{t}}$ respectively. Then, (3.14) and Prop. 3.2 give

$$
R_{i_{1}, \ldots, i_{t}}=a_{i_{1}, \ldots, i_{t}}+\sum_{r=1}^{t-2} \overline{\left(\int_{z}^{\mathfrak{a}} F_{i_{1}, \ldots, i_{r}}\right)} R_{i_{r+1}, \ldots, i_{t}} .
$$

For convenience we have set $Z_{j}:=f_{j}$. (3.30) and induction imply that

$$
\left.Z_{i_{1}, \ldots, i_{t}}\right|_{2}\left(\gamma_{1}-1\right) \ldots\left(\gamma_{t-1}-1\right)=\overline{\int_{\mathfrak{a}}^{\gamma_{1}^{-1} \mathfrak{a}} f_{i_{1}}} \ldots \overline{\int_{\mathfrak{a}}^{\gamma_{t-1}^{-1} \mathfrak{a}} f_{i_{t-1}} f_{i_{t}}}
$$

In the third step, we suitably modify the functions constructed so far to obtain holomorphic forms. To state a lemma we will need, we recursively define the following functions:

$$
\begin{gathered}
S_{i_{1}}:=\overline{\int_{z}^{\mathfrak{a}} f_{i_{1}}(w) d w} \text { and } \\
S_{i_{1}, \ldots, i_{t}}:=\sum_{r=1}^{t} \overline{\int_{z}^{\mathfrak{a}} F_{i_{1}, \ldots, i_{r}}(w) d w} S_{i_{r+1}, \ldots, i_{t} .} .
\end{gathered}
$$

We also set $S_{i_{k+1}, \ldots, i_{k}}=1, S_{i_{j}, \ldots, i_{k}}=0$ for $j>k+1$ and $S_{i_{0}}=1$.

Lemma 3.8. Let $t \in \mathbb{Z}_{\geq 2}$. For every $i_{1}, \ldots, i_{t} \in\{1, \ldots, g\}$ we have (i) For $m \neq 0$,

$$
R_{i_{1}, \ldots, i_{t}}=\sum_{j=0}^{t-2} S_{i_{1}, \ldots, i_{j}} \cdot a_{i_{j+1}, \ldots, i_{t}} .
$$

(ii)

$$
\operatorname{Res}_{s=1} Z_{\mathfrak{a} 0}\left(\cdot, s, 1,0 ; \bar{F}_{i_{1}, \ldots, i_{t}}\right)=\frac{1}{V} S_{i_{1}, \ldots, i_{t}} .
$$


Proof: (i) is proved by induction in $t$. By (3.14), it is clear for $t=2$. If the result holds for orders $<t$, then (3.31) implies that

$$
R_{i_{1}, \ldots, i_{t}}=a_{i_{1}, \ldots, i_{t}}+\sum_{r=1}^{t-2} \overline{\left(\int_{z}^{\mathfrak{a}} F_{i_{1}, \ldots, i_{r}}\right)} \sum_{j=r}^{t-2} S_{i_{r+1}, \ldots, i_{j}} a_{i_{j+1}, \ldots, i_{t}} .
$$

By the definition of $S_{i_{j} \ldots i_{k}}$ for $j>k$, the inner sum can be written in the form $\sum_{j=1}^{t-2} S_{i_{r+1}, \ldots, i_{j}} a_{i_{j+1}, \ldots, i_{t}}$ and thus

$$
\left.R_{i_{1}, \ldots, i_{t}}=a_{i_{1}, \ldots, i_{t}}+\sum_{j=1}^{t-2} \sum_{r=1}^{t-2} \overline{\left(\int_{z}^{\mathfrak{a}} F_{i_{1}, \ldots, i_{r}}\right.} S_{i_{r+1}, \ldots, i_{j}}\right) a_{i_{j+1}, \ldots, i_{t}} .
$$

Since $S_{i_{j}, \ldots, i_{k}}=0$ for $j>k+1$, the inner sum equals $S_{i_{1}, \ldots, i_{j}}$. This proves the identity for $t$.

(ii) follows from (3.14), Prop. 3.2 and a straightforward induction argument because, as shown in the proof of Prop. 3.6., $a_{i_{1}, \ldots, i_{t}}$ in (3.14) is 0 .

By (3.29) and Lemma 3.8(i), there is a linear combination $Z\left(i_{1}, \ldots, i_{t-1}\right)$ of $Z_{i_{1}, i_{2}}, \ldots, Z_{i_{1}, \ldots, i_{t-1}}$ for $m \neq 0$ such that

$$
\frac{d}{d \bar{z}}\left(Z_{i_{1}, \ldots, i_{t}}-Z\left(i_{1}, \ldots, i_{t-1}\right)\right)=\frac{i}{2 y^{2}}\left(S_{i_{1}, \ldots, i_{t-2}} a_{i_{t-1}, i_{t}}\right)
$$

Since

$$
\operatorname{Res}_{s=1}\left(Q_{\mathfrak{a} m}\left(z, s, 1,0 ; \bar{F}_{i_{t-1}}\right)\right)=\frac{-\overline{a_{\mathfrak{a}}(m)}}{2 \pi i m}=2 \overline{<\overline{<f_{i_{t-1}}, P_{\mathfrak{a} m}(\cdot)>}}
$$

(where $\langle\cdot, \cdot\rangle$ is the usual Petersson scalar product), by the definition of $a_{i_{t-1}, i_{t}}$ we have,

$$
a_{i_{t-1}, i_{t}}=2 i \overline{<f_{i_{t-1}}, f_{i_{t}}>} .
$$

Therefore, if $i_{t-1} \neq i_{t},(3.33)$ and the orthonormality of the basis imply that $Z_{i_{1}, \ldots, i_{t}}-Z\left(i_{1}, \ldots, i_{t-1}\right)$ is holomorphic. If $i_{t-1}=i_{t},(3.33)$ again implies that

$$
\left(Z_{i_{1}, \ldots, i_{t}}-Z\left(i_{1}, \ldots, i_{t-1}\right)\right)-\left(Z_{i_{1}, \ldots, i_{t-2}, 1,1}-Z\left(i_{1}, \ldots, i_{t-2}, 1\right)\right)
$$

is holomorphic.

In order to keep track of the basis elements we will construct in the sequel, we indicate the conjugation of $\overline{\int_{i \infty}^{\gamma_{j} i \infty} f_{i_{j}}(w) d w}$ by a minus sign in the notation of the corresponding subscript. Specifically, in view of $(3.32)$ we set, for every $i_{1}, \ldots, i_{t} \in\{1, \ldots, g\}$ with $\left(i_{t-1}, i_{t}\right) \neq(1,1)$,

$$
\mathcal{Z}_{-i_{1}, \ldots,-i_{t-1}, i_{t}}= \begin{cases}(-1)^{t-1}\left(Z_{i_{1}, \ldots, i_{t}}-Z\left(i_{1}, \ldots, i_{t-1}\right)\right) & \text { if } i_{t-1} \neq i_{t} \\ (-1)^{t-1}\left(Z_{i_{1}, \ldots, i_{t}}-Z\left(i_{1}, \ldots, i_{t-1}\right)-Z_{i_{1}, \ldots, 1,1}-Z\left(i_{1}, \ldots, i_{t-2}, 1\right)\right) & \text { if } i_{t-1}=i_{t} .\end{cases}
$$

The reason we have added the factor $(-1)^{t-1}$ is so that $\mathcal{Z}$ satisfy (3.32) without the $\gamma_{j}$ 's being inverted on the right-hand side. For $t=1$, we set, for $i<0$,

$$
\mathcal{Z}_{i}=\mathcal{Z}_{-i}=f_{i}:=f_{-i}
$$

Theorem 3.9. For $t \in \mathbb{Z}_{\geq 2}, i_{1}, \ldots, i_{t-1} \in\{-1, \ldots,-g\}, i_{t} \in\{1, \ldots, g\}$ and $\left(i_{t-1}, i_{t}\right) \neq(-1,1)$, we have $\mathcal{Z}_{i_{1}, \ldots, i_{t}} \in S_{2}^{t}$.

Proof: They are holomorphic by construction. The invariance under the parabolic elements of all $Z_{i_{1}, \ldots, i_{r}}$ 's is deduced by (3.30) and the fact that $F_{i_{1}, \ldots, i_{r}} \in S_{2}^{t}$. (3.32) implies that

$$
\left.\mathcal{Z}_{i_{1}, \ldots, i_{t}}\right|_{2}\left(\gamma_{1}-1\right) \ldots\left(\gamma_{t}-1\right)=0 \text {. }
$$


The growth condition proved in Theorem 3.4 implies that each $Z_{i_{1}, \ldots, i_{r}}$ and thus $\mathcal{Z}_{i_{1}, \ldots, i_{t}}$ is $\ll$ $y_{\mathfrak{F}}(z)^{-1 / 2}$. (Recall that $Z_{\mathfrak{a} m}(z, s+1,1,2 ; \bar{f})$ is divided by $y$ in $Z_{\mathfrak{a} m}(z, s ; \bar{f})$ ). Considering the Fourier expansion of $\mathcal{Z}_{i_{1}, \ldots, i_{t}}$ we deduce its vanishing at the cusps.

Now, (3.30) and Th. 3.4 imply that $\left.Z_{i_{1}, \ldots, i_{r}}\right|_{2} \gamma \ll y_{\mathfrak{F}}(z)^{-1 / 2}$ for each $\gamma \in \Gamma$. Therefore, the same estimate holds for the holomorphic $\left.\mathcal{Z}_{i_{1}, \ldots, i_{t}}\right|_{2} \gamma$ and a look at its Fourier expansion implies the vanishing at the cusps for each $\gamma$.

By (the second formulation of) the definition of $S_{2}^{t}$ we deduce the result.

In the next section we will also need a family of functions lying outside $S_{2}^{t}$. They are the analogue of the function $Z_{\mu, \mu}+2 i V \overline{<\mu, \mu>} P_{\mathfrak{a} 0}(z)_{2}$ in Prop. 5.2 of [DO]. If $\mathfrak{a}_{i}(i=1, \ldots, m)$ is a set of inequivalent cusps for $\Gamma$, set

$$
f_{g+i}:=P_{\mathfrak{a}_{i} 0}-P_{\mathfrak{a}_{m} 0} \quad \text { for } i=1, \ldots, m-1 .
$$

Then $\left\{f_{g+1}, \ldots, f_{g+m-1}\right\}$ a basis of the space $\mathcal{E}_{2}$ of Eisenstein series of $M_{2}$ (cf. [GO]). For $i_{j} \in$ $\{1, \ldots, g\}$, we set

$$
\mathcal{Z}_{-i_{1}, \ldots, i_{t-1}, i_{t}}^{\prime}= \begin{cases}\mathcal{Z}_{-i_{1}, \ldots, i_{t}} & \text { if } i_{t-1} \neq i_{t} \\ (-1)^{t-1}\left(Z_{i_{1}, \ldots, i_{t}}-Z\left(i_{1}, \ldots, i_{t-1}\right)-2 i V \cdot Z_{\mathfrak{a}_{m} 0}\left(\cdot, 0 ; \bar{F}_{i_{1}, \ldots, i_{t-2}}\right)\right) & \text { if } i_{t-1}=i_{t} .\end{cases}
$$

The last term is understood to be $P_{\mathfrak{a}_{m} 0}(\cdot)_{2}$ when $t=2$. By Lemma 3.8(ii), (3.29) and (3.33) we observe that $\mathcal{Z}_{-i_{1}, \ldots,-i_{t-1}, i_{t}}^{\prime}$ is holomorphic. By the holomorphicity, Theorem 3.4 and $(3.8), \mathcal{Z}_{-i_{1}, \ldots, i_{t}}^{\prime}$ vanishes at all cusps except $\mathfrak{a}_{m}$ in the case $t=2$. Again the reason for the factor $(-1)^{t-1}$ is that $\mathcal{Z}^{\prime}$ now satisfies (3.32) without the $\gamma_{j}$ 's being inverted on the right-hand side.

\subsection{Construction of a basis of $S_{2}^{t}$}

We will use the functions defined in Section 3.3 to build recursively bases for all $S_{2}^{t}$ 's. We first introduce some notation and prove an elementary lemma. First, following $[R]$, for an increasing finite sequence $j_{1}, \ldots j_{t-1}$ we call a shuffle of type $(r, t)$ a pair $(\phi, \psi)$ of order-preserving maps

$$
\phi:\left\{j_{1}, \ldots, j_{r-1}\right\} \rightarrow\{1, \ldots, t-1\} \quad \text { and } \psi:\left\{j_{r}, \ldots, j_{t-1}\right\} \rightarrow\{1, \ldots, t-1\}
$$

whose images are disjoint and complementary. Since the specific underlying sequence will be understood in each case, we denote their set simply by $\mathcal{S}_{r, t}$. We then have

Lemma 3.10. For $F \in S_{2}^{r}, G \in M_{0}^{t-r+1}$ we have

$$
\left.(F \cdot G)\right|_{2}\left(\gamma_{1}-1\right) \ldots\left(\gamma_{t-1}-1\right)=\left.\left.\sum_{(\phi, \psi) \in \mathcal{S}_{r, t}} F\right|_{2}\left(\gamma_{\phi(1)}-1\right) \ldots\left(\gamma_{\phi(r-1)}-1\right) \cdot G\right|_{0}\left(\gamma_{\psi(r)}-1\right) \ldots\left(\gamma_{\psi(t-1)}-1\right) .
$$

Proof: By Th. 2.2 of $[\mathrm{CD}]$, we have

$$
\left.(F \cdot G)\right|_{2}(\gamma-1)=\left.F\right|_{2}(\gamma-1) \cdot G+\left.F \cdot G\right|_{0}(\gamma-1)+\left.\left.F\right|_{2}(\gamma-1) \cdot G\right|_{0}(\gamma-1) .
$$

This means that each time we apply a $\gamma-1(\gamma \in \Gamma)$ on $F \cdot G$, either $F$ or $G$ or both are acted upon by $\gamma-1$ too. Now, $F$ (resp. $G$ ) is annihilated by any products with $r$ (resp. $t-r+1$ ) factors of the form $\gamma-1$. Therefore, the only non-vanishing terms left after $\left(\gamma_{1}-1\right) \ldots\left(\gamma_{t-1}-1\right)$ is applied on $F \cdot G$ are the products of the form

$$
\left.\left.F\right|_{2}\left(\gamma_{i_{1}}-1\right) \ldots\left(\gamma_{i_{r-1}}-1\right) \cdot G\right|_{0}\left(\gamma_{j_{1}}-1\right) \ldots\left(\gamma_{j_{t-r}}-1\right)
$$

with $i_{1}<\cdots<i_{r-1}, j_{1}<\cdots<j_{t-r}$ and $i_{1}, \ldots, i_{r-1}, j_{1}, \ldots, j_{t-r}$ covering $\{1, \ldots, t-1\}$. In particular, by the last two facts the sets of $i_{k}$ and $j_{k}$ are disjoint. This implies Lemma 3.10. 
Next, for $j \in\{ \pm 1, \ldots, \pm g\} \cup\{g+1, \ldots, g+m-1\}$ we set $<f_{j}, \gamma>$ for $\int_{i}^{\gamma i} f_{j}(w) d w$, when $j>0$ and $\overline{\int_{i \infty}^{\gamma i \infty} f_{-j}(w) d w}$, for $j<0$. We also set $\mathcal{A}$ for the space generated by maps $\phi: \Gamma^{l} \rightarrow S_{2}$ defined by

$$
\phi\left(\gamma_{1}, \ldots, \gamma_{l}\right)=<f_{i_{1}}, \gamma_{1}>\cdots<f_{i_{l}}, \gamma_{l}>f_{i_{l+1}}
$$

with $-i_{j}=i_{j+1}=1$ for at least one $j \in\{1, \ldots, l\}$. (In section 4 we will need the analogue of this space with $f_{i_{l+1}}$ of higher weight. In an effort to simplify notation, $\mathcal{A}$ will stand for the space in the case of weight 2 and when we need it for higher weights, we will indicate it by a subscript.)

We denote by $I^{\prime}$ the set of vectors $\left(i_{1}, \ldots, i_{t}\right)$ with entries in $\{ \pm 1, \ldots, \pm g\}$ for which there is no $j \in\{1, \ldots, t-1\}$ such that $-i_{j}=i_{j+1}=1$. We also let $I$ be the set of $\left(i_{1}, \ldots, i_{t}\right) \in I^{\prime}$, with $i_{t}>0$.

Starting with $\mathcal{Z}_{i}$, suppose now that, for each $s<t$, the forms $\mathcal{Z}_{i_{1}, \ldots, i_{s}},\left(i_{1}, \ldots, i_{s}\right) \in I$ satisfy

$$
\left.\mathcal{Z}_{i_{1}, \ldots, i_{s}}\right|_{2}\left(\gamma_{1}-1\right) \ldots\left(\gamma_{s-1}-1\right)=<f_{i_{1}}, \gamma_{1}>\cdots<f_{i_{s-1}}, \gamma_{s-1}>f_{i_{s}}+\phi\left(\gamma_{1}, \ldots, \gamma_{s-1}\right)
$$

for some $\phi \in \mathcal{A}$.

We claim that for every $\left(i_{1}, \ldots, i_{t}\right) \in I$, there are $\mathcal{Z}_{i_{1}, \ldots, i_{t}}$ satisfying (3.34) with $s=t$.

First, with Lemma 3.10,

$$
\begin{aligned}
& {\left.\left[\mathcal{Z}_{i_{1}} \int_{i}^{z} \mathcal{Z}_{i_{2}, \ldots, i_{t}}(w) d w\right]\right|_{2}\left(\gamma_{1}-1\right) \ldots\left(\gamma_{t-1}-1\right)=} \\
& f_{i_{1}}<f_{i_{2}}, \gamma_{1}>\cdots<f_{i_{t-1}}, \gamma_{t-2}>\int_{i}^{\gamma_{t-1} i} f_{i_{t}}(w) d w+\phi\left(\gamma_{1}, \ldots, \gamma_{t-1}\right) .
\end{aligned}
$$

Hence, for $\left(i_{1}, \ldots, i_{t-1}\right) \in I$ and $i_{t} \in\{1, \ldots, g\}$ we set

$$
\mathcal{Z}_{i_{1}, \ldots, i_{t}}:=\mathcal{Z}_{i_{t}} \int_{i}^{z} \mathcal{Z}_{i_{1}, \ldots, i_{t-1}}(w) d w
$$

Next, if $i_{1}<0,\left(i_{1}, i_{2}\right) \in I$, and $\left(i_{3}, \ldots, i_{t}\right) \in I$,

$$
\begin{aligned}
& {\left.\left[\mathcal{Z}_{i_{1}, i_{2}} \int_{i}^{z} \mathcal{Z}_{i_{3}, \ldots, i_{t}}(w) d w\right]\right|_{2}\left(\gamma_{1}-1\right) \ldots\left(\gamma_{t-1}-1\right)=} \\
& \sum_{(\phi, \psi) \in \mathcal{S}_{2, t}} \overline{\int_{i}^{\gamma_{\phi(1)} i} f_{i_{1}}(w) d w} f_{i_{2}} \prod_{j=3}^{t-1}<f_{i_{j}}, \gamma_{\psi(j)}>\int_{i}^{\gamma_{\psi(t)} i} f_{i_{t}}(w) d w+\phi\left(\gamma_{1}, \ldots, \gamma_{t-1}\right)
\end{aligned}
$$

for some $\phi \in \mathcal{A}$. By the definition of shuffles, $\int_{i}^{\gamma_{t-1} i} f_{i_{t}}(w) d w$ appears as the last factor and thus unconjugated in each summand of the right-hand side except for that corresponding to $\phi(1)=t-1$. Therefore, by (3.35), there is a linear combination of $\mathcal{Z}_{j_{1}} \int_{i}^{z} \mathcal{Z}_{j_{2}, \ldots, j_{t}}(w) d w$ 's denoted by $A\left(f_{i_{1}}, \ldots, f_{i_{t}}\right)$, such that

$$
\begin{aligned}
& {\left.\left[\mathcal{Z}_{i_{1}, i_{2}} \int_{i}^{z} \mathcal{Z}_{i_{3}, \ldots, i_{t}}(w) d w-A\left(f_{i_{1}}, \ldots, f_{i_{t}}\right)\right]\right|_{2}\left(\gamma_{1}-1\right) \ldots\left(\gamma_{t-1}-1\right)=} \\
& \overline{\int_{i}^{\gamma_{t-1} i} f_{i_{1}}(w) d w} f_{i_{2}}<f_{i_{3}}, \gamma_{1}>\cdots<f_{i_{t-1}}, \gamma_{t-3}>\int_{i}^{\gamma_{t-2} i} f_{i_{t}}(w) d w+\phi\left(\gamma_{1}, \ldots, \gamma_{t-1}\right)
\end{aligned}
$$

for some $\phi \in \mathcal{A}$. Hence for $\left(i_{t-1}, i_{t}\right),\left(i_{1}, \ldots, i_{t-2}\right) \in I$ and $i_{t-1}<0$ we can set

$$
\mathcal{Z}_{i_{1}, \ldots, i_{t}}:=\mathcal{Z}_{i_{t-1}, i_{t}} \int_{i}^{z} \mathcal{Z}_{i_{1}, \ldots, i_{t-2}}(w) d w-A\left(f_{i_{t-1}}, f_{i_{t}}, f_{i_{1}}, \ldots, f_{i_{t-2}}\right) .
$$


Further, if $i_{1}, i_{2}<0$ and $\left(i_{1}, i_{2}, i_{3}\right),\left(i_{4}, \ldots, i_{t}\right) \in I$,

$$
\begin{aligned}
& {\left.\left[\mathcal{Z}_{i_{1}, i_{2}, i_{3}} \int_{i}^{z} \mathcal{Z}_{i_{4}, \ldots, i_{t}}(w) d w\right]\right|_{2}\left(\gamma_{1}-1\right) \ldots\left(\gamma_{t-1}-1\right) } \\
& \sum_{(\phi, \psi) \in \mathcal{S}_{3, t}} \frac{\int_{i}^{\gamma_{\phi(1)} i} f_{i_{1}}(w) d w \int_{i}^{\gamma_{\phi(2)} i} f_{i_{2}}(w) d w}{f_{i_{3}}} \prod_{j=4}^{t-1}<f_{i_{j}}, \gamma_{\psi(j)}>\int_{i}^{\gamma_{\psi(t)} i} f_{i_{t}}(w) d w+ \\
& \phi\left(\gamma_{1}, \ldots, \gamma_{t-1}\right)
\end{aligned}
$$

for some $\phi \in \mathcal{A}$. The only summand that does not appear on the right-hand side of (3.35) and (3.36) for an appropriate permutation of $i_{1}, \ldots, i_{t}$, is

$$
\overline{\int_{i}^{\gamma_{t-2} i} f_{i_{1}}(w) d w \int_{i}^{\gamma_{t-1} i} f_{i_{2}}(w) d w} f_{i_{3}}<f_{i_{4}}, \gamma_{1}>\cdots \int_{i}^{\gamma_{t-3} i} f_{i_{t}}(w) d w .
$$

Therefore, by (3.35) and (3.36), there is a linear combination of terms $\mathcal{Z}_{j_{1}} \int_{i}^{z} \mathcal{Z}_{j_{2}, \ldots, j_{t}}(w) d w$ 's and $\mathcal{Z}_{j_{1}, j_{2}} \int_{i}^{z} \mathcal{Z}_{j_{3}, \ldots, j_{t}}(w) d w$ 's denoted by $B\left(f_{i_{1}}, \ldots, f_{i_{t}}\right)$, such that

$$
\begin{aligned}
& {\left.\left[\mathcal{Z}_{i_{1}, i_{2}, i_{3}} \int_{i}^{z} \mathcal{Z}_{i_{4}, \ldots, i_{t}}(w) d w-B\left(f_{i_{1}}, \ldots, f_{i_{t}}\right)\right]\right|_{2}\left(\gamma_{1}-1\right) \ldots\left(\gamma_{t-1}-1\right)=} \\
& \overline{\int_{i}^{\gamma_{t-2} i} f_{i_{1}}(w) d w \int_{i}^{\gamma_{t-1} i} f_{i_{2}}(w) d w} f_{i_{3}}<f_{i_{4}}, \gamma_{1}>\cdots \int_{i}^{\gamma_{t-3} i} f_{i_{t}}(w) d w+\phi\left(\gamma_{1}, \ldots, \gamma_{t-1}\right)
\end{aligned}
$$

for some $\phi \in \mathcal{A}$. Hence for $\left(i_{t-2}, i_{t-1}, i_{t}\right),\left(i_{1}, \ldots, i_{t-3}\right) \in I$ and $i_{t-2}, i_{t-1}<0$ we can set

$$
\mathcal{Z}_{i_{1}, \ldots, i_{t}}:=\mathcal{Z}_{i_{t-2}, i_{t-1}, i_{t}} \int_{i}^{z} \mathcal{Z}_{i_{1}, \ldots, i_{t-3}}(w) d w-B\left(f_{i_{t-2}}, f_{i_{t-1}}, f_{i_{t}}, f_{i_{1}}, \ldots, f_{i_{t-3}}\right) .
$$

Continuing this way we construct, for all $\left(i_{1}, \ldots, i_{t}\right) \in I$, functions $\mathcal{Z}_{i_{1}, \ldots i_{t}}$ satisfying (3.34). The last element, whose indices $i_{1}, \ldots, i_{t-1}$ are negative, is obtained directly from Th. 3.9.

We also define recursively a similar family of functions involving $\mathcal{Z}^{\prime}$ : Starting with $\mathcal{Z}_{i}^{\prime}=\mathcal{Z}_{i}$, suppose that, for each $s<t, \mathcal{Z}_{i_{1}, \ldots, i_{s}}^{\prime}, i_{j} \in\{ \pm 1, \cdots \pm g\}\left(i_{s}>0\right)$ satisfy

$$
\left.\mathcal{Z}_{i_{1}, \ldots, i_{s}}^{\prime}\right|_{2}\left(\gamma_{1}-1\right) \ldots\left(\gamma_{s-1}-1\right)=<f_{i_{1}}, \gamma_{1}>\cdots<f_{i_{s-1}}, \gamma_{s-1}>f_{i_{s}} .
$$

Then

$$
\left.\left[\mathcal{Z}_{i_{1}}^{\prime} \int_{i}^{z} \mathcal{Z}_{i_{2}, \ldots, i_{t}}^{\prime}(w) d w\right]\right|_{2}\left(\gamma_{1}-1\right) \ldots\left(\gamma_{t-1}-1\right)=f_{i_{1}}<f_{i_{2}}, \gamma_{1}>\cdots<f_{i_{t-1}}, \gamma_{t-2}>\int_{i}^{\gamma_{t-1} i} f_{i_{t}}(w) d w
$$

and for $i_{j} \in\{ \pm 1, \cdots \pm g\}\left(i_{t-1}>0\right)$ we set

$$
\mathcal{Z}_{i_{1}, \ldots, i_{t}}^{\prime}:=\mathcal{Z}_{i_{t}}^{\prime} \int_{i}^{z} \mathcal{Z}_{i_{1}, \ldots, i_{t-1}}^{\prime}(w) d w
$$

Next, if $i_{1}<0$,

$$
\begin{aligned}
& {\left.\left[\mathcal{Z}_{i_{1}, i_{2}}^{\prime} \int_{i}^{z} \mathcal{Z}_{i_{3}, \ldots, i_{t}}^{\prime}(w) d w\right]\right|_{2}\left(\gamma_{1}-1\right) \ldots\left(\gamma_{t-1}-1\right)=} \\
& \sum_{(\phi, \psi) \in \mathcal{S}_{2, t}} \overline{\int_{i}^{\gamma_{\phi(1)} i} f_{i_{1}}(w) d w} f_{i_{2}} \prod_{j=3}^{t-1}<f_{i_{j}}, \gamma_{\psi(j)}>\int_{i}^{\gamma_{\psi(t)} i} f_{i_{t}}(w) d w .
\end{aligned}
$$


As before, there is a linear combination of $\mathcal{Z}_{j_{1}}^{\prime} \int_{i}^{z} \mathcal{Z}_{j_{2}, \ldots, j_{t}}^{\prime}(w) d w$ 's denoted by $A\left(f_{i_{1}}, \ldots, f_{i_{t}}\right)$, such that

$$
\begin{aligned}
{\left.\left[\mathcal{Z}_{i_{1}, i_{2}}^{\prime} \int_{i}^{z} \mathcal{Z}_{i_{3}, \ldots, i_{t}}^{\prime}(w) d w-A\left(f_{i_{1}}, \ldots, f_{i_{t}}\right)\right]\right|_{2}\left(\gamma_{1}-1\right) \ldots\left(\gamma_{t-1}-1\right) } & = \\
& \frac{\int_{i}^{\gamma_{t-1} i} f_{i_{1}}(w) d w f_{i_{2}}<f_{i_{3}}, \gamma_{1}>\cdots<f_{i_{t-1}}, \gamma_{t-3}>\int_{i}^{\gamma_{t-2} i} f_{i_{t}}(w) d w}{}
\end{aligned}
$$

so, if $i_{t-1}<0$ we set

$$
\mathcal{Z}_{i_{1}, \ldots, i_{t}}^{\prime}:=\mathcal{Z}_{i_{t-1}, i_{t}}^{\prime} \int_{i}^{z} \mathcal{Z}_{i_{1}, \ldots, i_{t-2}}^{\prime}(w) d w-A\left(f_{i_{t-1}}, f_{i_{t}}, f_{i_{1}}, \ldots, f_{i_{t-2}}\right) .
$$

Continuing in this way we construct, for all $i_{j} \in\{ \pm 1, \ldots, \pm g\}\left(i_{t}>0\right)$, functions $\mathcal{Z}_{i_{1}, \ldots i_{t}}^{\prime}$ satisfying (3.34), but, this time, without a $\phi$. The last element, whose indices $i_{1}, \ldots, i_{t-1}$ are negative, is obtained directly by the construction at the end of section 3.3 .

By construction these functions are holomorphic and satisfy the stated functional equation. They have at most polynomial growth at each cusp $\mathfrak{a}$, and vanish at $\mathfrak{a} \neq \mathfrak{a}_{m}$. Because of this, they are invariant under $\pi_{\mathfrak{a}}$ for $\mathfrak{a} \neq \mathfrak{a}_{m}$.

To prove that the $\mathcal{Z}$ 's span $S_{2}^{t}$, we will need a lemma that generalizes Prop. 5.2. of [DO].

Lemma 3.11. Let $t \geq 3$. Suppose that for some $F \in S_{2}^{t}$ and $c_{i_{3}, \ldots, i_{t}} \in \mathbb{C}$,

$$
\left.F\right|_{2}\left(\gamma_{1}-1\right) \ldots\left(\gamma_{t-1}-1\right)=\sum c_{i_{3}, \ldots, i_{t}} \overline{\int_{i}^{\gamma_{1} i} f_{1}(w) d w} \int_{i}^{\gamma_{2} i} f_{1}(w) d w<f_{i_{3}}, \gamma_{3}>\ldots f_{i_{t}}
$$

for all $\gamma_{i} \in \Gamma$, where the sum ranges over all $i_{j} \in\{ \pm 1, \ldots, \pm g\}$. Then all $c_{i_{3}, \ldots, i_{t}}$ vanish.

Proof: As shown above,

$$
\left.\mathcal{Z}_{-1,1, \ldots, i_{t}}^{\prime}\right|_{2}\left(\gamma_{1}-1\right) \ldots\left(\gamma_{t-1}-1\right)=\overline{\int_{i}^{\gamma_{1} i} f_{1}(w) d w} \int_{i}^{\gamma_{2} i} f_{1}(w) d w<f_{i_{3}}, \gamma_{3}>\ldots f_{i_{t}}
$$

and hence $F-\sum c_{i_{3}, \ldots, i_{t}} \mathcal{Z}_{-1,1, \ldots, i_{t}}^{\prime}$ is annihilated by $\left(\gamma_{1}-1\right) \ldots\left(\gamma_{t-1}-1\right)$. Since $F$ and $\mathcal{Z}_{-1,1, \ldots}^{\prime}$ are of at most polynomial growth at the cusps, for every $\gamma_{i} \in \Gamma$, we have

$$
\left.\left(F-\sum c_{i_{3}, \ldots, i_{t}} \mathcal{Z}_{-1,1, \ldots, i_{t}}^{\prime}\right)\right|_{2}\left(\gamma_{1}-1\right) \ldots\left(\gamma_{t-2}-1\right)=\sum_{i=1}^{g+m-1} \chi_{i}\left(\gamma_{1}, \ldots, \gamma_{t-2}\right) f_{i}
$$

for $\chi_{i}: \Gamma^{t-2} \rightarrow \mathbb{C}$. The left-hand side is annihilated upon the application of one more $\gamma-1$ and hence the identity $\gamma \delta-1=(\gamma-1)(\delta-1)+(\gamma-1)+(\delta-1)$ implies that each $\chi_{i}$ is a group homomorphism in terms of each $\gamma_{j}$.

By Eichler-Shimura isomorphism,

$$
\chi_{i}\left(\gamma_{1}, \ldots, \gamma_{t-2}\right)=\sum_{j}\left(a_{j}^{i}\left(\gamma_{1}, \ldots, \gamma_{t-3}\right) \int_{i}^{\gamma_{t-2} i} h_{j}(w) d w+b_{j}^{i}\left(\gamma_{1}, \ldots, \gamma_{t-3}\right) \overline{\int_{i}^{\gamma_{t-2} i}} g_{j}(w) d w\right)
$$

for some $a_{j}^{i}, b_{j}^{i}: \Gamma^{t-3} \rightarrow \mathbb{C}, g_{j} \in S_{2}$ and $h_{j} \in M_{2}$. The injectivity of the Eichler-Shimura isomorphism implies that each $a_{j}^{i}, b_{j}^{i}$ is a homomorphism on each of the arguments and hence, by induction, we deduce that

$$
\begin{aligned}
& \left(F-\sum c_{i_{3}, \ldots, i_{t}} \mathcal{Z}_{-1,1, \ldots, i_{t}}^{\prime}\right) \mid\left(\gamma_{1}-1\right) \ldots\left(\gamma_{t-2}-1\right)= \\
& \qquad \sum_{i_{1}, \ldots, i_{t-1}} \lambda_{i_{1}, \ldots, i_{t-1}}<f_{i_{1}}, \gamma_{1}>\cdots<f_{i_{t-2}}, \gamma_{t-2}>f_{i_{t-1}}
\end{aligned}
$$


with $i_{j} \in\{ \pm 1, \ldots, \pm g\} \cup\{g+1, \ldots, g+m-1\}$ and $i_{t-1}>0$. Since $\mathcal{Z}_{i_{1}, \ldots, i_{t}}^{\prime}$ is invariant under $\pi_{\mathfrak{a}_{i}}$ when $i \neq m$, the identity $(\gamma-1)(\pi-1)=\left(\gamma \pi \gamma^{-1}-1\right) \gamma-\pi-1$ implies that

$$
\left.\mathcal{Z}_{i_{1}, \ldots, i_{t}}^{\prime}\right|_{2}\left(\gamma_{1}-1\right) \ldots\left(\gamma_{t-2}-1\right)
$$

vanishes when one of the $\gamma_{j}$ 's equals $\pi_{\mathfrak{a}_{i}}, i \neq m$. Since $F \in S_{2}^{t}$, this then also holds for both sides of (3.37). Therefore, none of the $f_{i_{j}}, j<t-1$ appearing in (3.37) can be non-cuspidal. Indeed, for $0<k<m,<f_{i_{j}}, \pi_{\mathfrak{a}_{k}}>\neq 0$ iff $i_{j}=g+k$ (recall the definition of $f_{g+1}$ ). Hence, for all $\gamma_{1}, \ldots, \gamma_{j-1}, \gamma_{j+1}, \ldots, \gamma_{t-2} \in \Gamma$ and for $\gamma_{j}=\pi_{\mathfrak{a}_{k}},(k=1, \ldots, m-1)$, the RHS of (3.37) equals

$$
\begin{aligned}
& \sum_{i_{1}, \ldots, i_{j-1}, i_{j+1}, \ldots, i_{t-1}} \lambda_{i_{1}, \ldots, i_{j-1}, g+k, i_{j+1}, \ldots i_{t-1}} \times \\
& \quad<f_{i_{1}}, \gamma_{1}>\cdots<f_{i_{j-1}}, \gamma_{j-1}><f_{g+k}, \pi_{\mathfrak{a}_{k}}><f_{i_{j+1}}, \gamma_{j+1}>\cdots<f_{i_{t-2}}, \gamma_{t-2}>f_{i_{t-1}}=0 .
\end{aligned}
$$

Therefore, by the injectivity of Eichler-Shimura isomorphism, $\lambda_{i_{1}, \ldots, i_{j-1}, g+k, i_{j+1}, i_{t-1}}=0$. Hence, only cusp forms $f_{j}$ appear on the RHS of (3.37), so if $\left\{\gamma_{1}, \ldots, \gamma_{t-2}\right\}$ contains $\pi_{\mathfrak{a}_{m}}$, both sides of (3.37) vanish. Since $F \in S_{2}^{t}$, this implies that $\left.\sum c_{i_{3}, \ldots, i_{t}} \mathcal{Z}_{-1,1, \ldots, i_{t}}^{\prime}\right|_{2}\left(\gamma_{1}-1\right) \ldots\left(\gamma_{t-2}-1\right)=0$ if at least one of the $\gamma_{i}$ 's is $\pi_{\mathfrak{a}_{m}}$. However, we can show, by induction that, when $i_{1}<0$,

$$
\left.\mathcal{Z}_{i_{1}, i_{2}, \ldots, i_{t}}^{\prime}\right|_{2}\left(\pi_{\mathfrak{a}_{m}}-1\right) \ldots\left(\gamma_{t-2}-1\right)=f_{i_{t}}<f_{i_{t-1}}, \gamma_{t-2}>\cdots \int_{i}^{\pi_{\mathfrak{a}_{m}} i} \mathcal{Z}_{-i, i}^{\prime}(w) d w
$$

if $\left(i_{1}, i_{2}\right)=(-i, i)(i>0)$ and 0 otherwise. For $t=3$, it is straightforward. If $i_{r}>0, i_{r+1}, \ldots, i_{t-1}<0$, then $\mathcal{Z}_{-i, i, i_{3}, \ldots}^{\prime}$ is by definition equal to $\mathcal{Z}_{i_{r+1} \ldots i_{t}}^{\prime} \int_{i}^{z} \mathcal{Z}_{-i, i, i_{3}, \ldots, i_{r}}^{\prime}$ minus a linear combination of products of the form $\mathcal{Z}_{i_{r+k}, \ldots, i_{t}}^{\prime} \int_{i}^{z} \mathcal{Z}_{j_{1}, j_{2}, j_{3}, \ldots}^{\prime}(k>1)$ which we denote by $C\left(f_{-i}, f_{i}, f_{i_{3}}, \ldots\right)$. The indices $j_{1}, j_{2}, \ldots$ in the expression of $C\left(f_{i_{1}}, f_{i_{2}}, \ldots\right)$ are obtained by interspersing, in their original order, subsets of $\left\{i_{r+1}, \ldots, i_{t-1}\right\}$ among the indices $i_{1}, i_{2}, i_{3}, \ldots, i_{t}$. Now, as in Lemma 3.10 , we observe that, if one of the $\gamma_{i}$ is parabolic and $i_{r+k}, \ldots i_{t-1}<0, i_{r+k-1}>0$ then, for $k \geq 1$,

$$
\begin{aligned}
{\left[\mathcal{Z}_{i_{r+k} \ldots i_{t}}^{\prime}\right.} & \left.\int_{i}^{z} \mathcal{Z}_{j_{1}, j_{2}, \ldots, i_{r+k-1}}^{\prime}\right]\left.\right|_{2}\left(\gamma_{1}-1\right) \ldots\left(\gamma_{t-2}-1\right)= \\
& \left.\sum_{(\phi, \psi) \in \mathcal{S}} \mathcal{Z}_{i_{r+k}, \ldots i_{t}}^{\prime}\right|_{2}\left(\gamma_{\phi(1)}-1\right) \ldots\left(\gamma_{\phi(t-r-k)}-1\right) \times \\
& \int_{z}^{\gamma_{\psi(r+k-2)} z}\left[\left.\mathcal{Z}_{j_{1}, j_{2}, \ldots, i_{r+k-1}}^{\prime}\right|_{2}\left(\gamma_{\psi(1)}-1\right) \ldots\left(\gamma_{\psi(r+k-3)}-1\right)\right](w) d w .
\end{aligned}
$$

Therefore, by the second part of the inductive hypothesis, the application of $\left(\pi_{\mathfrak{a}_{m}}-1\right) \ldots\left(\gamma_{t-2}-1\right)$ on $\mathcal{Z}_{-i, i, \ldots}^{\prime}$ will eliminate all terms in $C\left(f_{-i}, f_{i}, f_{i_{3}}, \ldots\right)$ except for those with $j_{1}=-i, j_{2}=i$, thus obtaining, by (the first part of) the inductive hypothesis, products of the form

$$
\left(\int_{i}^{\gamma_{\psi(1)} i} \mathcal{Z}_{-i, i}^{\prime}(w) d w\right) \cdots<f_{i_{r+k-1}}, \gamma_{\psi(r+k-2)}><f_{i_{r+k}}, \gamma_{\phi(1)}>\cdots<f_{i_{t-1}}, \gamma_{\phi(t-r-k)}>f_{i_{t}} .
$$

These, upon varying $k$, yield

$$
\begin{array}{r}
\sum_{(\phi, \psi) \in \mathcal{S}_{r, t-1}}\left(\int_{i}^{\gamma_{\psi(1)} i} \mathcal{Z}_{-i, i}^{\prime}(w) d w\right) \cdots<f_{i_{r}}, \gamma_{\psi(r-1)}><f_{i_{r+1}}, \gamma_{\phi(1)}>\cdots<f_{i_{t-1}}, \gamma_{\phi(t-r-1)}>f_{i_{t}}+ \\
\left.C\left(\mathcal{Z}_{-i, i}^{\prime}, \ldots, f_{i_{t}}\right)\right|_{2}\left(\gamma_{1}-1\right) \ldots\left(\gamma_{t-2}-1\right) .
\end{array}
$$

where $C\left(\mathcal{Z}_{-i, i}^{\prime}, f_{i_{3}}, \ldots\right)$ is $C\left(f, f_{i_{3}}, \ldots\right)$ with $f$ formally replaced by $\mathcal{Z}_{-i, i}^{\prime}$. Since the cancellations yielding (3.34') do not rely on $f_{i_{1}}$ 's being a cusp form rather than second order modular form, the 
same cancellations imply that $(3.39)$ equals $f_{i_{t}}<f_{i_{t-1}}, \gamma_{t-2}>\cdots \int_{i}^{\pi_{\mathfrak{a}_{m}}{ }^{i}} \mathcal{Z}_{-i, i}^{\prime}(w) d w$ as we wanted to show.

The second part of the claim is proved in the same way, once we observe that $C\left(f_{i_{1}}, f_{i_{2}}, \ldots\right)$ does not contain integrals of $\mathcal{Z}_{-i, i, \ldots}^{\prime}$ because of the way the indices $j_{1}, j_{2}, \ldots$ in the expression of $C\left(f_{i_{1}}, f_{i_{2}}, \ldots\right)$ are obtained and the fact that $i_{r+1}, \ldots, i_{t-1}<0$. Therefore the inductive hypothesis can be applied.

Therefore, for all $\gamma_{2}, \ldots, \gamma_{t-2} \in \Gamma$,

$$
\begin{aligned}
\left.\sum c_{i_{3}, \ldots, i_{t}} \mathcal{Z}_{-1,1, \ldots, i_{t}}^{\prime}\right|_{2}\left(\pi_{\mathfrak{a}_{m}}-1\right) \ldots\left(\gamma_{t-2}-1\right)= & \\
& \left(\int_{i}^{\pi_{\mathfrak{a}_{m}} i} \mathcal{Z}_{-1,1}^{\prime}(w) d w\right) \sum c_{i_{3}, \ldots, i_{t}}<f_{i_{3}}, \gamma_{2}>\cdots<f_{i_{t-1}}, \gamma_{t-2}>f_{i_{t}}
\end{aligned}
$$

and since the LHS is 0 , the linear independence of the terms $<f_{i_{3}}, \gamma_{2}>\cdots<f_{i_{t-1}}, \gamma_{t-2}>f_{i_{t}}$ for $\gamma_{2}, \ldots, \gamma_{t-2} \in \Gamma$, implies the desired conclusion.

Theorem 3.12. Let $t \geq 1$. Then the image of

$$
\left\{\mathcal{Z}_{i_{1}, \ldots, i_{t}} ;\left(i_{1}, \ldots, i_{t}\right) \in I\right\}
$$

under the natural projection is a basis of $S_{2}^{t} / S_{2}^{t-1}$.

Proof: We first note that by Th. 3.1 of $[\mathrm{CD}]$, each $\mathcal{Z}_{i_{1}, \ldots, i_{t}}\left(\left(i_{1}, \ldots, i_{t}\right) \in I\right)$ is a weight 2 , $t$-th order form cusp because it is the product ("0-th Rankin-Cohen bracket") of a weight 2, order $r<t$ cusp form and a weight 0 , order $t-r+1$ modular form (namely the antiderivative of a weight 2 , order $t-r$ cusp form).

We now show that our set spans $S_{2}^{t} / S_{2}^{t-1}$. The claim is obvious for $t=1$. Let now $t>1$ and $F \in S_{2}^{t}$. Then, for every $\gamma_{i} \in \Gamma$,

$$
\left.F\right|_{2}\left(\gamma_{1}-1\right) \ldots\left(\gamma_{t-1}-1\right)=\sum_{i} \chi_{i}\left(\gamma_{1}, \ldots, \gamma_{t-1}\right) f_{i}
$$

for a $\chi_{i}: \Gamma^{t-1} \rightarrow \mathbb{C}$. As in the proof of Lemma 3.11, each $\chi_{i}$ is a group homomorphism but, in addition, each of them vanishes at the parabolic elements as a function of each $\gamma_{i}$. Repeated applications of the Eichler-Shimura isomorphism imply that each $\chi_{i}\left(\gamma_{1}, \ldots, \gamma_{t-1}\right)$ is a linear combination of

$$
\prod_{j=1}^{t-1}<f_{i_{j}}, \gamma_{j}>\quad i_{j} \in\{ \pm 1, \ldots, \pm g\}
$$

By the construction of $\mathcal{Z}_{i_{1}, \ldots i_{t}}$ 's and (3.34) (with $s=t$ ) we then deduce that there is a linear combination $L$ of these functions satisfying, $\left.(F-L)\right|_{2}\left(\gamma_{1}-1\right) \ldots\left(\gamma_{t-1}-1\right)=\phi\left(\gamma_{1}, \ldots, \gamma_{t-1}\right)$ for some $\phi \in \mathcal{A}$. We will show that $\phi \equiv 0$.

By the definition of $\mathcal{A}$, there are $c_{i_{1}, \ldots, \hat{i}_{j}, \hat{i}_{j+1}, \ldots, i_{t}} \in \mathbb{C}$ such that $\phi\left(\gamma_{1}, \ldots, \gamma_{t-1}\right)$ equals

$$
\sum_{j=1}^{t-1} \sum c_{i_{1}, \ldots, \hat{i}_{j}, \hat{i}_{j+1}, \ldots, i_{t}}<f_{i_{1}}, \gamma_{1}>\ldots \overline{\bar{\int}_{i}^{\gamma_{j} i} f_{1}(w) d w} \int_{i}^{\gamma_{j+1} i} f_{1}(w) d w<f_{i_{j+2}}, \gamma_{j+2}>\ldots f_{i_{t}}
$$

for all $\gamma_{i} \in \Gamma$. The inner sum ranges over all $\left(i_{1}, \ldots, \hat{i}_{j}, \hat{i}_{j+1}, \ldots, i_{t}\right)$ such that $\left(i_{j+2} \ldots, i_{t}\right) \in I$ and the hat indicates missing index. The term corresponding to $j=t-1$ is understood to end with $\overline{\int_{i}^{\gamma_{t-1} i} f_{1}(w) d w} f_{1}$. With (3.34), an induction shows that, for some $a_{i_{1}, \ldots}^{l}, b_{i_{j+2}, \ldots}^{l} \in \mathbb{C}$, the sum can be re-written as

$$
\begin{aligned}
& \sum_{j=1}^{t-1} \sum_{l} \sum_{0<\left|i_{k}\right| \leq g} a_{i_{1}, \ldots, i_{j-1}}^{l}<f_{i_{1}}, \gamma_{1}>\cdots \times \\
& \frac{\int_{i}^{\gamma_{j} i} f_{1}(w) d w}{\int_{i}^{\gamma_{j+1} i}} f_{1}(w) d w \sum_{0<\left|i_{k}\right| \leq g} b_{i_{j+2}, \ldots, i_{t-1}}^{l}<f_{i_{j+2}}, \gamma_{j+2}>\ldots f_{i_{t}}
\end{aligned}
$$


with

$$
\int_{i}^{\gamma_{j+1} i} f_{1}(w) d w \sum_{0<\left|i_{k}\right| \leq g} b_{i_{j+2}, \ldots, i_{t-1}}^{l}<f_{i_{j+2}}, \gamma_{j+2}>\ldots f_{i_{t}}=\left.G\right|_{2}\left(\gamma_{j+1}-1\right) \ldots\left(\gamma_{t-1}-1\right)
$$

for some $G \in S_{2}^{t-j}$.

This implies that, if we consider $\gamma_{1}, \ldots, \gamma_{t-2}$ fixed for the time being, each term in (3.40) except for that corresponding to $j=t-1$ is a multiple of a $\left.G\right|_{2}\left(\gamma_{t-1}-1\right)$ for some $G \in S_{2}^{2}$. Hence, if $\left.(F-L)\right|_{2}\left(\gamma_{1}-1\right) \ldots\left(\gamma_{t-1}-1\right)=\phi\left(\gamma_{1}, \ldots, \gamma_{t-1}\right)$, then there is a second-order form $G_{1}$ and a $\mu_{t-1} \in \mathbb{C}$ (which will normally depend on $\gamma_{1}, \ldots, \gamma_{t-2}$ ) such that

$$
\left.G_{1}\right|_{2}\left(\gamma_{t-1}-1\right)=\mu_{t-1} \overline{\int_{i \infty}^{\gamma_{t-1} i \infty} f_{1}(w) d w} f_{1}
$$

Prop. 5.2 of $[\mathrm{DO}]$ implies that $\mu_{t-1}=0$. Therefore, $\phi\left(\gamma_{1}, \ldots, \gamma_{t-1}\right)$ equals the expression in (3.40) but with one term less.

We can continue the 'descent' this way by noting each term on the right-hand side except for the last one is a multiple of a $\left.G\right|_{2}\left(\gamma_{t-2}-1\right)\left(\gamma_{t-1}-1\right)$ for some $G \in S_{2}^{3}$. Therefore, there is a $G_{2} \in S_{2}^{3}$ such that

$$
\left.G_{2}\right|_{2}\left(\gamma_{t-2}-1\right)\left(\gamma_{t-1}-1\right)=\sum c_{i_{t}} \overline{\int_{i \infty}^{\gamma_{t-2} i \infty} f_{1}(w) d w} \int_{i \infty}^{\gamma_{t-1} i \infty} f_{1}(w) d w f_{i_{t}}
$$

for some $c_{i_{t}} \in \mathbb{C}$ and this, from Lemma 3.11, implies that all $c_{i_{t}}$ vanish. Continuing this way we deduce that $\phi \equiv 0$.

We will finally show that the $\mathcal{Z}$ 's are linearly independent modulo $S_{2}^{t-1}$. Suppose that

$$
\sum_{\left(i_{1}, \ldots, i_{t}\right) \in I} \lambda_{i_{1}, \ldots, i_{t}} \mathcal{Z}_{i_{1}, \ldots, i_{t}}=0
$$

Then

$$
\left.\sum_{\left(i_{1}, \ldots, i_{t}\right) \in I} \lambda_{i_{1}, \ldots, i_{t}} \mathcal{Z}_{i_{1}, \ldots, i_{t}}\right|_{2}\left(\gamma_{1}-1\right) \ldots\left(\gamma_{t-1}-1\right)=0
$$

and by (3.34) (with $s=t$ ), we obtain

$$
\sum_{\left(i_{1}, \ldots, i_{t}\right) \in I} \lambda_{i_{1}, \ldots, i_{t}}<f_{i_{1}}, \gamma_{1}>\cdots<f_{i_{t-1}}, \gamma_{t-1}>f_{i_{t}}+\phi\left(\gamma_{1}, \ldots, \gamma_{t-1}\right)=0,
$$

for some $\phi \in \mathcal{A}$. Since the set of all $\chi_{i}$ 's is linearly independent and $\phi$ is a linear combination of $<f_{i_{1}}, \gamma_{1}>\cdots<f_{i_{t-1}}, \gamma_{t-1}>f_{i_{t}}$ 's with $\left(i_{1}, \ldots, i_{t}\right) \notin I$, we deduce that $\lambda_{i_{1}, \ldots, i_{t}}=0$, for all $\left(i_{1}, \ldots, i_{t}\right) \in I$, i.e. the $\mathcal{Z}$ 's are linearly independent.

Corollary 3.13. Let $g$ be the genus of $\Gamma$. The dimension of $S_{2}^{t}(\Gamma) / S_{2}^{t-1}(\Gamma)$ ( $\left.t \geq 1\right)$ is 0 if $g=0$, and

$$
\frac{1}{2}\left(\left(g+\sqrt{g^{2}-1}\right)^{t}+\left(g-\sqrt{g^{2}-1}\right)^{t}\right)
$$

otherwise.

Proof: According to the theorem, to prove the corollary in the case $g \neq 0$, it suffices to compute the cardinality $a_{t}$ of $I$. If $b_{t}$ is the cardinality of $I^{\prime}$, then, $b_{t}-a_{t}$ is the number of vectors in $I^{\prime}$ with $i_{t}<0$. In particular, $i_{t} \neq 1$ and hence, $b_{t}-a_{t}=g b_{t-1}$. On the other hand, $b_{t}=2 g b_{t-1}-b_{t-2}$. $\left(g b_{t-1}\right.$ elements of $I^{\prime}$ have $i_{t}<0$ and $g b_{t-1}-b_{t-2}$ have $i_{t}>0$ with $\left.\left(i_{t-1}, i_{t}\right) \neq(-1,1)\right)$. The solution of this recursive relation with $b_{1}=2 g, b_{2}=4 g^{2}-1$ is

$$
b_{t}=\frac{1}{2 \sqrt{g^{2}-1}}\left(\left(g+\sqrt{g^{2}-1}\right)^{t+1}-\left(g-\sqrt{g^{2}-1}\right)^{t+1}\right)
$$


when $g \neq 1$ and $b_{t}=t+1$, when $g=1$. The conclusion then follows from $b_{t}-a_{t}=g b_{t-1}$.

The proof of the corollary in the case $g=0$ follows by the observation that, for $F \in S_{2}^{t}(\Gamma)$,

$$
\left.F\right|_{2}\left(\gamma_{1}-1\right) \ldots\left(\gamma_{t-1}-1\right) \in S_{2}(\Gamma)
$$

Since $S_{2}(\Gamma)=\{0\}$ when $g=0$, this implies that $F \in S_{2}^{t-1}(\Gamma)$ and hence $S_{2}^{t}(\Gamma) / S_{2}^{t-1}(\Gamma)=\{0\}$.

Examples (with $g \neq 0$ ):

$$
\begin{array}{cc}
t & \operatorname{dim}\left(S_{2}^{t}(\Gamma) / S_{2}^{t-1}(\Gamma)\right) \\
1 & g \\
2 & 2 g^{2}-1 \\
3 & 4 g^{3}-3 g \\
4 & 8 g^{4}-8 g^{2}+1 \\
5 & 16 g^{5}-20 g^{3}+5 g
\end{array}
$$

\section{Higher Weights}

Our construction of the base of $S_{k}^{t}$ for $k>2$ and $t>1$ relies on the base for $S_{2}^{t-1}$ we have just defined and it parallels the process we employed to construct the latter. We shall maintain the notation developed in the previous sections.

First of all we note that Theorem 3.4 can be used just as well to construct forms of higher weight. Specifically, for every $k>2$ and $f \in A_{t}$ we set

$$
\begin{aligned}
Y_{\mathfrak{a} m}(z, s ; \bar{f}) & :=y^{-k / 2} Z_{\mathfrak{a} m}(z, s+k / 2,1, k ; \bar{f}) \\
& =\sum_{\gamma \in \Gamma_{\mathfrak{a}} \backslash \Gamma} \overline{\left(\int_{\mathfrak{a}}^{\gamma \mathfrak{a}} f(w) d w\right)} \operatorname{Im}\left(\sigma_{\mathfrak{a}}{ }^{-1} \gamma z\right)^{s} e\left(m \sigma_{\mathfrak{a}}{ }^{-1} \gamma z\right) j\left(\sigma_{\mathfrak{a}}{ }^{-1} \gamma, z\right)^{-k} .
\end{aligned}
$$

According to Theorem 3.4, it is analytic for $\operatorname{Re}(s)>1-k / 2-\delta_{\Gamma}$ and it is easy to see that

$$
\begin{aligned}
& \left.Y_{\mathfrak{a} m}\left(\cdot, 0, \bar{F}_{i_{1}, \ldots, i_{t-1}}\right)\right|_{k}(\gamma-1)= \\
& \overline{\left(\int_{\mathfrak{a}}^{\gamma^{-1} \mathfrak{a}} F_{i_{1}, \ldots, i_{t-1}}\right)} P_{\mathfrak{a} m}(\cdot)_{k}+\sum_{r=1}^{t-2} \overline{\left(\int_{\mathfrak{a}}^{\gamma^{-1} \mathfrak{a}} F_{i_{1}, \ldots, i_{r}}\right)} Y_{\mathfrak{a} m}\left(\cdot, 0, \bar{F}_{i_{r+1}, \ldots, i_{t-1}}\right)
\end{aligned}
$$

Here we set

$$
P_{\mathfrak{a} m}(z)_{k}:=y^{-1} U_{\mathfrak{a} m}(z, 1, k),
$$

the Poincaré series of weight $k$. There is a set of positive integers $M$ such that $\left\{P_{\mathfrak{a} m}(\cdot)_{k}\right\}_{m \in M}$ is a basis of $S_{k}$.

Now, for $\operatorname{Re}(s)$ large we have:

$$
\frac{d}{d \bar{z}} Y_{\mathfrak{a} m}\left(z, s ; \bar{F}_{i_{1}, \ldots, i_{t-1}}\right)=\frac{i s}{2 y^{1+k / 2}} Z_{\mathfrak{a} m}\left(z, s+k / 2,1, k-2 ; \bar{F}_{i_{1}, \ldots, i_{t-1}}\right)
$$

By Theorem 3.4., $Z_{\mathfrak{a} m}\left(z, s+k / 2,1, k-2 ; \bar{F}_{i_{1}, \ldots, i_{t-1}}\right)$ is holomorphic at $s=0$, when $k>2$ and hence $Y_{\mathfrak{a} m}\left(z, 0 ; \bar{F}_{i_{1}, \ldots, i_{t-1}}\right)$ is holomorphic in $z$. Thus we set $\mathcal{Y}_{m}:=P_{\mathfrak{a} m}(\cdot)_{k}$ and

$$
\mathcal{Y}_{-i_{1}, \ldots,-i_{t-1} ; m}=(-1)^{t-1} Y_{\mathfrak{a} m}\left(z, 0 ; \bar{F}_{i_{1}, \ldots, i_{t-1}}\right) \text {. }
$$

With (4.1) we note that

$$
\left.\mathcal{Y}_{-i_{1}, \ldots,-i_{t-1} ; m}\right|_{k}\left(\gamma_{1}-1\right) \ldots\left(\gamma_{t-1}-1\right)=\overline{\int_{\mathfrak{a}}^{\gamma_{1} \mathfrak{a}} f_{i_{1}}} \ldots \overline{\int_{\mathfrak{a}}^{\gamma_{t-1} \mathfrak{a}} f_{i_{t-1}}} P_{\mathfrak{a} m}(\cdot)_{k} .
$$


In exactly the same way as Th. 3.9 , we can show that $\mathcal{Y}_{i_{1}, \ldots, i_{t} ; m} \in S_{k}^{t}$ for $i_{j} \in\{-1, \ldots,-g\}$.

We can now construct a basis for $S_{k}^{t+1}$ by extending the definition of $\mathcal{Y}_{i_{1}, \ldots, i_{t} ; m}$ to all $\left(i_{1}, \ldots, i_{t}\right) \in I^{\prime}$ and $m>0$ : First, with Lemma 3.10, for $\left(i_{1}, \ldots, i_{t}\right) \in I$

$$
\begin{aligned}
& {\left.\left[\mathcal{Y}_{m}(z) \int_{i}^{z} \mathcal{Z}_{i_{1}, \ldots, i_{t}}(w) d w\right]\right|_{k}\left(\gamma_{1}-1\right) \ldots\left(\gamma_{t}-1\right) }= \\
& P_{\mathfrak{a} m}(\cdot)_{k}<f_{i_{1}}, \gamma_{1}>\cdots<f_{i_{t-1}}, \gamma_{t-1}>\int_{i}^{\gamma_{t} i} f_{i_{t}}(w) d w+\phi\left(\gamma_{1}, \ldots, \gamma_{t}\right)
\end{aligned}
$$

for some $\phi \in \mathcal{A}_{k}$. Hence, for $\left(i_{1}, \ldots, i_{t}\right) \in I$ we set

$$
\mathcal{Y}_{i_{1}, \ldots, i_{t} ; m}:=\mathcal{Y}_{m}(z) \int_{i}^{z} \mathcal{Z}_{i_{1}, \ldots, i_{t}}(w) d w .
$$

Next, if $i_{1}<0$, and $\left(i_{2}, \ldots, i_{t}\right) \in I$,

$$
\begin{aligned}
& {\left.\left[\mathcal{Y}_{i_{1} ; m}(z) \int_{i}^{z} \mathcal{Z}_{i_{2}, \ldots, i_{t}}(w) d w\right]\right|_{k}\left(\gamma_{1}-1\right) \ldots\left(\gamma_{t}-1\right)=} \\
& \sum_{(\phi, \psi) \in \mathcal{S}_{2, t+1}} \overline{\int_{i}^{\gamma_{\phi(1)} i} f_{i_{1}}(w) d w} P_{\mathfrak{a} m}(\cdot)_{k} \prod_{j=2}^{t}<f_{i_{j}}, \gamma_{\psi(j)}>\int_{i}^{\gamma_{\psi(t)} i} f_{i_{t}}(w) d w+\phi\left(\gamma_{1}, \ldots, \gamma_{t}\right)
\end{aligned}
$$

for some $\phi \in \mathcal{A}_{k}$. Therefore, as in Section 3.4, there is a linear combination of $\mathcal{Y}_{m}(z) \int_{i}^{z} \mathcal{Z}_{j_{1}, \ldots, j_{t}}(w) d w$ 's denoted by $A_{i_{1}, \ldots, i_{t} ; m}$, such that

$$
\begin{aligned}
& {\left.\left[\mathcal{Y}_{i_{1}, m}(z) \int_{i}^{z} \frac{\mathcal{Z}_{i_{2}, \ldots, i_{t}}(w) d w}{z}-A_{i_{1}, \ldots, i_{t} ; m}\right]\right|_{k}\left(\gamma_{1}-1\right) \ldots\left(\gamma_{t}-1\right)=} \\
& \int_{i}^{\gamma_{t} i} f_{i_{1}}(w) d w P_{\mathfrak{a} m}(\cdot)_{k}<f_{i_{2}}, \gamma_{1}>\cdots<f_{i_{t-1}}, \gamma_{t-2}>\int_{i}^{\gamma_{t-1} i} f_{i_{t}}(w) d w+\phi\left(\gamma_{1}, \ldots, \gamma_{t}\right)
\end{aligned}
$$

for some $\phi \in \mathcal{A}_{k}$. Hence for $\left(i_{1}, \ldots, i_{t-1}\right) \in I$ and $i_{t}<0$ we can set

$$
\mathcal{Y}_{i_{1}, \ldots, i_{t} ; m}(z):=\mathcal{Y}_{i_{t} ; m}(z) \int_{i}^{z} \mathcal{Z}_{i_{1}, \ldots, i_{t-1}}(w) d w-A_{i_{t}, i_{1}, \ldots, i_{t-1} ; m} .
$$

Further, if $i_{1}, i_{2}<0$ and $\left(i_{3}, \ldots, i_{t}\right) \in I$,

$$
\begin{aligned}
& {\left[\mathcal{Y}_{i_{1}, i_{2} ; m}(z) \int_{i}^{z}\right.}\left.\mathcal{Z}_{i_{3}, \ldots, i_{t}}(w) d w\right]||_{k}\left(\gamma_{1}-1\right) \ldots\left(\gamma_{t}-1\right)= \\
& \sum_{(\phi, \psi) \in \mathcal{S}_{3, t+1}} \int_{i}^{\gamma_{\phi(1)} i} f_{i_{1}}(w) d w \int_{i}^{\gamma_{\phi(2)} i} f_{i_{2}}(w) d w P_{\mathfrak{a} m}(\cdot)_{k} \prod_{j=3}^{t}<f_{i_{j}}, \gamma_{\psi(j)}>\int_{i}^{\gamma_{\psi(t)}{ }^{i}} f_{i_{t}}(w) d w+ \\
& \phi\left(\gamma_{1}, \ldots, \gamma_{t}\right)
\end{aligned}
$$

for some $\phi \in \mathcal{A}_{k}$. Therefore, there is a linear combination of terms $\mathcal{Y}_{m}(z) \int_{i}^{z} \mathcal{Z}_{j_{1}, \ldots, j_{t}}(w) d w$ 's and $\mathcal{Y}_{j_{1} ; m}(z) \int_{i}^{z} \mathcal{Z}_{j_{2}, \ldots, j_{t}}(w) d w$ 's denoted by $B_{i_{1}, \ldots, i_{t} ; m}$, such that

$$
\frac{\left.\left[\mathcal{Y}_{i_{1}, i_{2} ; m}(z) \int_{i}^{z} \mathcal{Z}_{i_{3}, \ldots, i_{t}}(w) d w-B_{i_{1}, \ldots, i_{t} ; m}\right]\right|_{k}\left(\gamma_{1}-1\right) \ldots\left(\gamma_{t}-1\right)=}{\int_{i}^{\gamma_{t-1} i} f_{i_{1}}(w) d w \int_{i}^{\gamma_{t} i} f_{i_{2}}(w) d w P_{\mathfrak{a} m}(\cdot)_{k}<f_{i_{3}}, \gamma_{1}>\cdots \int_{i}^{\gamma_{t-2} i} f_{i_{t}}(w) d w+\phi\left(\gamma_{1}, \ldots, \gamma_{t}\right)}
$$

for some $\phi \in \mathcal{A}_{k}$. Hence for $\left(i_{1}, \ldots, i_{t-2}\right) \in I$ and $i_{t-2}, i_{t-1}<0$ we can set

$$
\mathcal{Y}_{i_{1}, \ldots, i_{t} ; m}(z):=\mathcal{Y}_{i_{t-1}, i_{t} ; m}(z) \int_{i}^{z} \mathcal{Z}_{i_{1}, \ldots, i_{t-2}}(w) d w-B_{i_{t-1}, i_{t}, i_{1}, \ldots, i_{t-2}} .
$$

Continuing this way we cover the entire $I^{\prime}$ and hence we construct, for all $\left(i_{1}, \ldots, i_{t}\right) \in I^{\prime}$, functions $\mathcal{Y}_{i_{1}, \ldots i_{t} ; m}$ such that

$$
\left.\mathcal{Y}_{i_{1}, \ldots, i_{t} ; m}\right|_{k}\left(\gamma_{1}-1\right) \ldots\left(\gamma_{t}-1\right)=<f_{i_{1}}, \gamma_{1}>\cdots<f_{i_{t}}, \gamma_{t}>P_{\mathfrak{a} m}(\cdot)_{k}+\phi\left(\gamma_{1}, \ldots, \gamma_{t}\right)
$$

for some $\phi \in \mathcal{A}_{k}$. 
Theorem 4.1. If $k \geqslant 4$ and $g$ is the genus of $\Gamma$, then the image of the set

$$
\left\{\mathcal{Y}_{i_{1}, \ldots, i_{t} ; m} ;\left(i_{1}, \ldots, i_{t}\right) \in I^{\prime} ; m \in M\right\}
$$

under the natural projection is a basis of $S_{k}^{t+1}(\Gamma) / S_{k}^{t}(\Gamma)$. Therefore the dimension of $S_{k}^{t+1}(\Gamma) / S_{k}^{t}(\Gamma)$ is 0 if $g=0,(t+1) \operatorname{dim}\left(S_{k}(\Gamma)\right)$ if $g=1$, and

$$
\frac{\operatorname{dim}\left(S_{k}(\Gamma)\right)}{2 \sqrt{g^{2}-1}}\left(\left(g+\sqrt{g^{2}-1}\right)^{t+1}-\left(g-\sqrt{g^{2}-1}\right)^{t+1}\right)
$$

otherwise.

Proof: Each $\mathcal{Y}_{i_{1}, \ldots, i_{t} ; m}$ is a $(t+1)$-th order cusp form as the product of an order $r<t+1$ cusp form and an order $t+2-r$ modular form.

Now, since the argument does not depend on the weight, we can show exactly as in the proof of Th. 3.12, that if $F \in S_{k}^{t+1}$, then

$$
\left.F\right|_{k}\left(\gamma_{1}-1\right) \ldots\left(\gamma_{t}-1\right)=\sum_{i_{1}, \ldots, i_{t+1}} \lambda_{i_{1}, \ldots, i_{t}}<f_{i_{1}}, \gamma_{1}>\cdots<f_{i_{t}}, \gamma_{t}>F_{i_{t+1}} .
$$

Here $F_{i}$ ranges over a basis of $S_{k}$. By (4.2) we then conclude that there is a linear combination $L$ of functions in the set under consideration such that

$$
\left.(F-L)\right|_{k}\left(\gamma_{1}-1\right) \ldots\left(\gamma_{t}-1\right)=\phi\left(\gamma_{1}, \ldots, \gamma_{t}\right)
$$

for some $\phi \in \mathcal{A}_{k}$. It is easy to see that Lemma 3.11 holds in weights $>2$. The only adjustment required in the proof is that the construction of the analogue of $\mathcal{Z}^{\prime}$ is based on

$$
\mathcal{Y}_{i_{1}, \ldots, i_{r} ; m} \int_{i}^{z} \mathcal{Z}_{i_{r+1}, \ldots, i_{t}}^{\prime}
$$

instead of $\mathcal{Z}_{i_{1}, \ldots, i_{r}}^{\prime} \int_{i}^{z} \mathcal{Z}_{i_{r+1}, \ldots, i_{t}}^{\prime}$. We deduce that $\phi \equiv 0$ and hence, our set spans $S_{k}^{t+1} / S_{k}^{t}$.

The proof that the set is linearly independent is deduced directly from (4.2), exactly as in Th. 3.12.

The formula for the dimension in the case $g>0$ is deduced by the formula for $b_{t}:=\# I^{\prime}$ established in Cor. 3.13. In the case $g=0$, the dimension is 0 because of (4.3).

Examples (with $g \neq 0$ ):

$$
\begin{array}{cc}
t & \operatorname{dim}\left(S_{k}^{t}(\Gamma) / S_{k}^{t-1}(\Gamma)\right) \\
2 & 2 g \operatorname{dim}\left(S_{k}(\Gamma)\right) \\
3 & \left(4 g^{2}-1\right) \operatorname{dim}\left(S_{k}(\Gamma)\right) \\
4 & \left(8 g^{3}-4 g\right) \operatorname{dim}\left(S_{k}(\Gamma)\right) \\
5 & \left(16 g^{4}-12 g^{2}+1\right) \operatorname{dim}\left(S_{k}(\Gamma)\right)
\end{array}
$$

Acknowledgments. The authors thank Paul Gunnells and Cormac O'Sullivan for several helpful comments. We are also indebted to the referee for a careful reading of the manuscript and for many useful suggestions.

\section{REFERENCES}

[CD] Y. Choie, N. Diamantis, Rankin-Cohen brackets on higher-order modular forms, Proceedings of the Bretton Woods workshop on Multiple Dirichlet series (AMS Proceedings of Symposia in Pure Mathematics), 2006.

[CDO] G. Chinta, N. Diamantis, C. O'Sullivan, Second order modular forms, Acta Arithmetica 103 (2002), 209-223.

[CO] G. Chinta, C. O'Sullivan, Non-holomorphic Poincaré series constructed from derivatives and antiderivatives of cusp forms and bounds on period polynomials (to appear). 
[DKMO] N. Diamantis, M. Knopp, G. Mason, C. O'Sullivan, L-functions of second-order cusp forms, Ramanujan Journal 12 (3) (2006), 327-347.

[DO] N. Diamantis, C. O'Sullivan,, The dimensions of spaces of holomorphic second-order automorphic forms and their cohomology (to appear).

[DS] N. Diamantis, R. Sreekantan,, Iterated integrals and higher order automorphic forms, Commentarii Mathematici Helvetici 81(2) (2006), 481-494.

[FW] D. Farmer, K. Wilson, Converse theorems assuming a partial Euler product, The Ramanujan Journal.

[GO] D. Goldfeld, C. O'Sullivan, Estimating additive character sums for Fuchsian groups, Ramanujan J. 7 (2003), 241-267.

[I1] H. Iwaniec, Spectral methods of automorphic forms, 2nd ed., vol. 53, Graduate studies in mathematics, Amer. Math. Soc., 2002.

[JO] J. Jorgenson, C. O'Sullivan., Convolution Dirichlet series and a Kronecker limit formula for second-order Eisenstein series, Nagoya Math J. 179 (2005), 1-56.

[KZ] P. Kleban, D. Zagier, Crossing probabilities and modular forms, J. Stat. Phys. 113 (2003), 431-454.

$[\mathrm{M}] \quad Y u$. Manin, Iterated integrals of modular forms and noncommutative modular symbols, Algebraic geometry and number theory, Progr. Math., 253 (2006), 565-597,.

[PR] Y. Petridis, M. S. Risager, Modular symbols have a normal distribution, GAFA 14 (5) (2004), $1013-1043$.

[R] R. Ree, Lie elements and an algebra associated with shuffles., Ann. of Math. (2) 68 (1958), 210-220.

[Sh] G. Shimura, Introduction to the Arithmetic Theory of Automorphic Functions, Princeton Univ. Press, 1971. 